\title{
WEAK DISPERSIVE ESTIMATES FOR SCHRÖDINGER EQUATIONS WITH LONG RANGE POTENTIALS
}

\author{
J. A. BARCELÓ, A. RUIZ, L. VEGA AND M. C. VILELA
}

\begin{abstract}
We prove some local smoothing estimates for the Schrödinger initial value problem with data in $L^{2}\left(\mathbb{R}^{d}\right), d \geq 2$ and a general class of potentials. In the repulsive setting we have to assume just a power like decay $(1+|x|)^{-\gamma}$ for some $\gamma>0$. Also attractive perturbations are considered. The estimates hold for all time and as a consequence a weak dispersion of the solution is obtained. The proofs are based on similar estimates for the corresponding stationary Helmholtz equation and Kato H-smooth theory.
\end{abstract}

\section{INTRODUCTION}

We consider Schrödinger Hamiltonians $-\Delta+V(x)$, with $V$ a real potential, $x \in$ $\mathbb{R}^{d}$ and $d \geq 2$. We study some dispersive estimates, also called local smoothing estimates, for solutions of the evolution initial value problem

$$
\left\{\begin{array}{l}
i \partial_{t} u-\Delta_{x} u+V(x) u=0, \quad(x, t) \in \mathbb{R}^{d} \times \mathbb{R} \quad d \geq 2 \\
u(x, 0)=u_{0}(x)
\end{array}\right.
$$

under different conditions on the potential $V$.

The first result is concerned with repulsive potentials. Our notion of repulsion involves conditions on the sign of $V$ and $\partial_{r} V$ (the radial derivative of $V$ ), and therefore is more restrictive to the one given for example in [1 for which just conditions on the positive part of $\partial_{r} V$ are assumed.

Theorem 1.1. Let $V$ be a real valued function satisfying the following conditions:

i) $V \geq 0$,

ii) There exists $\gamma>0$ such that:

- If $d>3$, we suppose that there exist $\eta>0$ such that

$$
\gamma V(x)+|x| \partial_{r} V(x) \leq(1-\eta) \frac{(d-1)(d-3)}{2|x|^{2}}
$$

- For $d=3$, we suppose that there exists $W(t) \geq 0$ on $(0, \infty)$ such that

$$
\sup _{|x|=t}\left\{\gamma V(x)+|x| \partial_{r} V(x)\right\} \leq W(t)
$$

Date: February 2008.

The first and second authors were supported by Spanish Grant MTM2005-07652-C02-01, and the third and fourth by Spanish Grant MTM2004-03029. 
and

$$
\int_{0}^{\infty} t W(t) d t<\frac{1}{2}
$$

Let $H=-\Delta+V$, then for $d \geq 3$, the Schrödinger operator $e^{i t H}$ satisfies the following estimates:

$$
\sup _{R>0} \frac{1}{R} \int_{B(0, R)} \int_{-\infty}^{\infty}\left|D^{\frac{1}{2}} e^{i t H} u_{0}(x)\right|^{2} d t d x \leq C\left\|u_{0}\right\|_{2}^{2},
$$

where, for $\alpha \in \mathbb{C}$, the operator $D^{\alpha}$ is defined by $\widehat{D^{\alpha}} f(\xi)=|\xi|^{\alpha} \widehat{f}(\xi)$.

We also have

$$
\sup _{R>0} \frac{1}{R} \int_{B(0, R)} V^{\frac{1}{2}}(x) \int_{-\infty}^{\infty}\left|e^{i t H} u_{0}(x)\right|^{2} d t d x \leq C\left\|u_{0}\right\|_{2}^{2} .
$$

Estimate (5), usually known as the local smoothing estimate (see [4, 19, 20] and [18), goes back to the work of Kato and Kruzhkov-Faminski in the context of KdV equations. Notice that here we obtain an estimate global in time.

In [3] it is proved that for $d \geq 3$ and potentials $V$ satisfying the conditions (2) and (3) with $\gamma=0$ and (4)

$$
\begin{aligned}
& \sup _{R>0} \frac{1}{R} \int_{B(0, R)} \int_{-\infty}^{\infty}\left|\nabla_{x} e^{i t H} u_{0}(x)\right|^{2} d t d x \\
& +\int_{\mathbb{R}^{d}}\left(\partial_{r} V\right)_{-}(x) \int_{-\infty}^{\infty}\left|e^{i t H} u_{0}(x)\right|^{2} d t d x \leq C\left\|u_{0}\right\|_{H^{1 / 2}\left(\mathbb{R}^{d}\right)}^{2},
\end{aligned}
$$

where $\left(\partial_{r} V\right)_{-}$is the negative part of $\partial_{r} V$. Notice that the estimate requires the initial datum to be in the non-homogeneous Sobolev space $H^{\frac{1}{2}}\left(\mathbb{R}^{d}\right)$ which means that from the point of view of small frequencies the result is not so strong. In a sense this can not be avoided because in [21] (see also [6]) it is proved that under some conditions on the potential the right hand side of (17) can be replaced by

$$
\left\|u_{0}\right\|_{\dot{H}_{V}^{1 / 2}}^{2}:=\left\|(-\Delta+V)^{1 / 4} u_{0}\right\|_{L^{2}\left(\mathbb{R}^{d}\right)}^{2},
$$

and that this is optimal. In our new result (5) we write the homogeneous derivative and therefore there is no difference of behaviour between low and high frequencies. We also want to mention that we answer affirmatively the question posed in Remark 4 of [3] for $L^{2}$ initial data ( see Remark 2.3 to be more precise).

The approach we follow in this paper is based also on multiplier technics but, as opposite to [3], the estimates for the evolution equation rely on estimates for the resolvent and the Kato' smoothing theory. In [1] and [3] potentials homogeneous of degree zero are allowed. However Theorem 1.1 does not apply in this case.

Some typical examples of potentials satisfying the conditions of Theorem 1.1 are, for $V_{\infty} \geq 0$ :

i) The potential $V(x)=\frac{V_{\infty}\left(\frac{x}{|x|}\right)}{|x|^{\gamma}}$ where $0<\gamma<2$ and the potential $V(x)=$ $\frac{c}{|x|^{2}}$ with small $c>0$.

ii) $V(x)=\frac{V_{\infty}\left(\frac{x}{|x|}\right)}{\left(1+|x|^{2}\right)^{\frac{\alpha}{2}}}$ with $V_{\infty}$ bounded and $0<\alpha$. 
In the case $V_{\infty}>c_{0}>0$ estimate (6) is particularly relevant. In fact, assume for instance $V(x)=1 /|x|^{\alpha}$ with $0<\alpha<2$. As a consequence (see [15, v.IV, page 147) we have that for $\delta>0$, the following estimate for the spectral projection operators holds

$$
\sup _{R>0,\|f\|_{L^{2}}=1} \frac{1}{R} \int_{B(0, R)}\left|P_{(0, \delta]} f(x)\right|^{2} \frac{d x}{|x|^{\frac{\alpha}{2}}} \leq C \delta,
$$

where $C$ is an absolute constant. A similar estimate in the free case would mean, roughly speaking, that the usual Fourier transform $\widehat{f}(\xi)$ behaves as $|\xi|^{\beta}$ for $\xi$ close to the origin, where

$$
\beta \geq-\frac{n}{2}+\frac{1}{2}\left(1-\frac{\alpha}{2}\right),
$$

if $0<\alpha<2$. Since not every function in $L^{2}\left(\mathbb{R}^{d}\right)$ satisfies this condition, the above inequality has to be understood as a special feature of the generalized Fourier transform associated to the potential $V$. It would be interesting to know if Strichartz estimate holds in this case. Recall that the existence of $x_{0}$ such that $V_{\infty}\left(\frac{x_{0}}{\left|x_{0}\right|}\right)=0$ implies that Strichartz estimates do not hold, see [7] and [14].

Our next result concerns with potentials which are not necessarily repulsive. We first consider the case where the radial variation of the attractive part is not too big. We have the following result.

Theorem 1.2. Let $V$ and $n$ be two real valued functions. Assume that $V$ satisfies the conditions i) and ii) of the Theorem [1.1, and $n$ satisfies:

iii) $n=n_{1}+n_{2}<0$ where $n_{1} \in L^{\infty}\left(\mathbb{R}^{d}\right)$ and $n_{2}$ is such that

$$
\int_{\mathbb{R}^{d}}\left|n_{2}(x)\right||g(x)|^{2} d x \leq c_{1} \int_{\mathbb{R}^{d}}|\nabla g(x)|^{2} d x,
$$

for some $c_{1}$ with $0<c_{1}<1$.

iv) Given $\rho>0$, take $j_{0} \in \mathbb{Z}$ such that $2^{j_{0}}<\rho \leq 2^{j_{0}+1}$, then

$$
\beta_{\rho}=\sum_{j \geq j_{0}}^{\infty} 2^{j+1} \sup _{x \in C_{j}} \frac{\nabla n(x) \cdot x}{|x||n(x)|}+\rho \sup _{x \in B(0, \rho)} \frac{\nabla n(x) \cdot x}{|x||n(x)|}
$$

$$
\text { satisfies } \begin{cases}\beta_{\rho}<\frac{1}{4} & d>3 \\ \beta_{\rho}+\int_{0}^{\infty} t W(t) d t<\frac{1}{2} & d=3,\end{cases}
$$$$
\text { where } C_{j}=\left\{x \in \mathbb{R}^{d}: 2^{j}<|x| \leq 2^{j+1}\right\}, \quad \forall j \in \mathbb{Z} \text {. }
$$

Let $H=-\Delta+V+n$, then for $d \geq 3$ and $\tau_{0}>0$, the Schrödinger operator $e^{i t H}$ satisfies the following estimates:

$$
\begin{gathered}
\sup _{R \geq \rho} \frac{1}{R} \int_{B(0, R)} \int_{-\infty}^{\infty}\left|D^{\frac{1}{2}} e^{i t H} \mathcal{P}_{\tau_{0}} u_{0}(x)\right|^{2} d t d x \leq C\left(\beta_{\rho}, \tau_{0}\right)\left\|u_{0}\right\|_{2}^{2}, \\
\tau \sup _{R \geq \rho} \frac{1}{R} \int_{B(0, R)} \int_{-\infty}^{\infty}\left|e^{i t H} \mathcal{P}_{\tau} u_{0}(x)\right|^{2} d t d x \leq C\left(\beta_{\rho}, \tau_{0}\right)\left\|u_{0}\right\|_{2}^{2} \quad \tau \geq \tau_{0},
\end{gathered}
$$

where $\mathcal{P}_{\tau}$ is the spectral projection operator associated with $[\tau, \infty)$. 
We observe that condition (9) remains true if $n(x)$ is changed into $\mu n(x)$ for any $\mu>0$. It also applies to functions homogeneous of degree zero. Typical examples of such $n(x)$ are as follows:

Take $\omega(x)=\mu\left(1-e^{g(\infty)-g(|x|)}\right)$, where $\mu>0$ and $g$ is a radial function such that

$$
0 \leq g^{\prime} \leq \frac{b}{(1+r)^{\gamma}} \quad \forall r \geq 0,
$$

with $\gamma>1$ and $b=b(\gamma)>0$ small enough. Under these conditions, we can apply Theorem 1.2 to $n=\omega-\mu$. As a consequence, we get estimates similar to (10) and (11) for $e^{i t H} \mathcal{P}_{(\mu, \infty)} u_{0}$ with $H=-\Delta+V+\omega$.

In the above example $\omega \leq 0$, therefore for $\mu$ big enough non trivial eigenvalues can be expected and in that case estimates (9) and (10) can not be extended to all $\tau$. Notice however than one would expect the estimate on the spectral projection to be true for $\mathcal{P}_{(\tau, \infty)}$ with $\tau>0$ and not just for $\mathcal{P}_{(\mu, \infty)}$. This motivates our final result where we use a compactness argument that follows from a uniqueness theorem due to Ikebe and Saito [8. Some extra assumptions are necessary, and in particular the result does not apply to potentials homogeneous of degree zero. An extra bonus is that we can consider the case $d \geq 2$. We prove the following theorem.

Theorem 1.3. Let $V_{1}$ and $V_{2}$ be two real valued functions satisfying that there exist two constants $a>0$ and $\gamma>0$ such that

$$
\begin{aligned}
\left|V_{1}\right|(x) & \leq \frac{a}{(1+|x|)^{\gamma}} \\
\left|V_{2}\right|(x) & \leq \frac{a}{(1+|x|)^{\gamma+1}}, \\
\partial_{r} V_{1}(x) & \leq \frac{a}{(1+|x|)^{\gamma+1}},
\end{aligned}
$$

and $H=-\Delta+V_{1}+V_{2}$.

Then for $d \geq 2, \tau_{0}>0$, and $\alpha>0$ the following estimates hold:

$$
\begin{gathered}
\int_{-\infty}^{\infty}\left\|D^{\frac{1}{2}} e^{i t H} \mathcal{P}_{\tau_{0}} u_{0}(x)\right\|_{L^{2}\left((1+|x|)^{-1-\alpha}\right)}^{2} d t \leq C_{1}\left(\tau_{0}\right)\left\|u_{0}\right\|_{2}^{2}, \\
\tau \int_{-\infty}^{\infty}\left\|e^{i t H} \mathcal{P}_{\tau} u_{0}(x)\right\|_{L^{2}\left((1+|x|)^{-1-\alpha}\right)}^{2} d t \leq C_{2}\left(\tau_{0}\right)\left\|u_{0}\right\|_{2}^{2}, \quad \tau \geq \tau_{0} .
\end{gathered}
$$

Consider as before $\omega(x)=\mu\left(e^{g(|x|)-g(\infty)}-1\right)$, where $\mu>0$ and $g$ is a radial function such that

$$
0 \leq g^{\prime} \leq \frac{c}{(1+r)^{\beta}} \quad \forall r \geq 0 .
$$

Then if $\beta>1, c>0$ and we put $V_{1}=\omega$ we can apply Theorem 1.3 As a consequence, we get estimates similar to (15) and (16) for $e^{i t H} \mathcal{P}_{(\tau, \infty)} u_{0}$ with $H=$ $-\Delta+\omega$ and $\tau>0$.

Notice that we can consider repulsive perturbations which are long range. In particular, we extend and improve some of the results in 9 . On the other hand we have to exclude the case $\tau_{0}=0$. This is obvious because we can consider potentials 
with decay as $\frac{C}{|x|^{2}}$, and therefore there can exist 0-eigenfunctions. Take for example $u(x)=\left(1+|x|^{2}\right)^{\lambda}$ with $\lambda<-d / 2$ and $V(x)=\frac{\Delta u}{u}$.

As it was pointed out before, the proofs of the above theorems are based on similar estimates for the corresponding stationary Helmholtz equations and the general Kato's argument on smoothing operators as in [10] and [16. Actually we prove the so called supersmoothing estimates which are stronger, see [10]. The procedure we use to obtain these stationary estimates are basically the multiplier method, see [11, [12] and [17. Similar estimates were obtained in [13].

We prove Theorem 1.1 in section 2, Theorem 1.2 in section 3 and Theorem 1.3 in section 4. For completeness we include Appendix 1 with some useful identities and Appendix 2 with some calculations that will be needed in the proof of Theorem 1.1 .

\section{Notation.}

We shall make use of the spaces introduced in [2] which are given by the following norms

$$
\|u\|_{X}^{2}=\sup _{R>0} \frac{1}{R} \int_{B(0, R)}|u(x)|^{2} d x
$$

We shall replace the norm in the corresponding predual space for the equivalent expression:

$$
\|f\|_{X^{*}}=\sum_{j \in \mathbb{Z}}\left(2^{j+1} \int_{C_{j}}|f(x)|^{2} d x\right)^{\frac{1}{2}} .
$$

Notice that

$$
\int_{\mathbb{R}^{d}} f(x) g(x) d x \leq C\|f\|_{X}\|g\|_{X^{*}}
$$

where $C$ is a positive constant depending only on $d$.

For any $\rho>0$ such that $2^{j_{0}}<\rho \leq 2^{j_{0}+1}$, we write

$$
\|u\|_{X_{\rho}}^{2}=\sup _{R \geq \rho} \frac{1}{R} \int_{B(0, R)}|u(x)|^{2} d x
$$

and

$$
\|f\|_{X_{\rho}^{*}}=\sum_{j \geq j_{0}}\left(2^{j+1} \int_{C_{j}}|f(x)|^{2} d x\right)^{\frac{1}{2}}+\left(\rho \int_{B(0, \rho)}|f(x)|^{2} d x\right)^{\frac{1}{2}} .
$$

In this case, we also have

$$
\int_{\mathbb{R}^{d}} u(x) f(x) d x \leq C\|u\|_{X_{\rho}}\|f\|_{X_{\rho}^{*}}
$$

\section{Repulsive potentials}

We start by proving a priori estimates for the resolvent of the operator $H$ and then we proceed to prove Theorem 1.1 


\subsection{Estimates for the resolvent.}

Theorem 2.1. Let $V$ be a real valued function satisfying the conditions of Theorem 1.1, and let $u$ be a solution of the equation

$$
-\Delta u+V(x) u \pm i \epsilon u-\tau u=f, \quad \epsilon \neq 0, x \in \mathbb{R}^{d}(d \geq 3) .
$$

Then, for any $\tau \in \mathbb{R}$, the following a priori estimate holds

$$
\begin{aligned}
& \|\nabla u\|_{X}^{2}+\max \{0, \tau\}\|u\|_{X}^{2}+(d-3) \int_{\mathbb{R}^{d}} \frac{|u(x)|^{2}}{|x|^{3}} d x+\int_{\mathbb{R}^{d}} \frac{V(x)}{|x|}|u(x)|^{2} d x \\
& +\sup _{R>0} \frac{1}{R^{3}} \int_{B(0, R)}|u(x)|^{2} d x+\chi(d) \int_{\mathbb{R}^{3}} \frac{W(|x|)}{|x|}|u(x)|^{2} d x \leq C\|f\|_{X^{*}}^{2},
\end{aligned}
$$

where $C$ is a constant independent of $\epsilon$ and $\tau, W$ is the function of Theorem 1.1 and $\chi(d)$ is defined by

$$
\chi(d)= \begin{cases}1 & \text { if } d=3 \\ 0 & \text { if } d \neq 3\end{cases}
$$

Remark 2.2. From estimate (18) follows

$$
\left\|\nabla R_{H}(\tau \pm i \epsilon) f\right\|_{X} \leq C\|f\|_{X^{*}}
$$

and

$$
\left\|R_{H}(\tau \pm i \epsilon) f\right\|_{L^{2}\left(\frac{V(x)}{|x|}\right)} \leq C\|f\|_{X^{*}},
$$

where $C$ is independent of $\epsilon \neq 0$ and $\tau$ and $R_{H}$ denotes the resolvent operator of $H=-\Delta+V$.

Proof. By a density argument we might assume the a priori condition $f \in L^{2}\left(\mathbb{R}^{d}\right)$.

The proof is based on the following estimates for the solution of (17):

$$
\begin{gathered}
\left\{\begin{array}{l}
\quad\|\nabla u\|_{X}^{2}+\int_{\mathbb{R}^{d}} \frac{V(x)}{|x|}|u(x)|^{2} d x+\sup _{R>0} \frac{1}{R^{3}} \int_{B(0, R)}|u(x)|^{2} d x \\
+(d-3) \int_{\mathbb{R}^{d}} \frac{|u(x)|^{2}}{|x|^{3}} d x+\chi(d) \int_{\mathbb{R}^{3}} \frac{W(|x|)}{|x|}|u(x)|^{2} d x \\
\leq C\|f\|_{X^{*}}^{2}+\frac{1}{4}(|\epsilon|+\max \{0, \tau\})\|u\|_{X}^{2}
\end{array}\right. \\
|\epsilon|\|u\|_{X}^{2} \leq\|f\|_{X^{*}}^{2}+\sup _{R>0} \frac{1}{R^{3}} \int_{B(0, R)}|u(x)|^{2} d x+\|\nabla u\|_{X}^{2} \\
+\left\|V^{\frac{1}{2}} u\right\|_{X}^{2}+(d-3) \int_{\mathbb{R}^{d}} \frac{|u(x)|^{2}}{|x|^{3}} d x+\chi(d) \int_{\mathbb{R}^{3}} \frac{W(|x|)}{|x|}|u(x)|^{2} d x \\
\leq C\|f\|_{X^{*}}^{2}+\frac{1}{2}|\epsilon|\|u\|_{X}^{2}, \quad \tau \geq 0
\end{gathered}
$$

Estimate (18) follows easily from the three above estimates, assuming that the terms in the left hand side are finite (this will be seen through the proofs of these 
estimates) in fact: for $\tau<0,(18)$ is a consequence of (22) and (23) and for $\tau \geq 0$, (18) is a consequence of (24) and (23).

Proof of (22). The basic estimates to obtain (22) are (108) and (106) in the appendix. The point is the choice of an appropriate radial function $\Phi_{R}(x) \equiv \Phi_{R}(|x|)$ in (108) so that its left hand side to be an upper bound of the left hand side of (22).

We start by performing some calculations of derivatives of such a radial function. We have:

$$
\nabla \bar{u}(x) \cdot D^{2} \Phi_{R}(x) \cdot \nabla u(x)=\Phi_{R}^{\prime \prime}(|x|)\left|\partial_{r} u(x)\right|^{2}+\frac{\Phi_{R}^{\prime}(|x|)}{|x|}\left|\partial_{\tau} u(x)\right|^{2},
$$

where $\partial_{r} u$ and $\partial_{\tau} u$ are respectively the radial and tangential parts of the derivative of $u$.

We also have

$$
-\nabla \Phi_{R}(x) \cdot \nabla V(x)=-\Phi_{R}^{\prime}(|x|) \partial_{r} V(x) .
$$

Roughly speaking, we want the quadratic form determined by $D^{2} \Phi_{R}(x)$ to be positive and the function $\frac{1}{4} \Delta^{2} \Phi_{R}(x)+\frac{1}{2} \nabla V(x) \cdot \nabla \Phi_{R}(x)$ to be nonpositive.

In the case of $d>3$ we proceed to find $\Phi_{R}$. We will use a combination of the classical Morawetz multiplier $|x|$, see [12, and the multiplier $\left(1+|x|^{2}\right)^{1 / 2}$ ( adapted to the Hemholtz equation). More precisely, we start by considering

$$
\Phi(x)=\left(1+|x|^{2}\right)^{1 / 2}+|x| .
$$

As above we identify $\Phi(x) \equiv \Phi(r)$ with $|x|=r$. Then we have

$$
\begin{gathered}
\Delta^{2} \Phi(x)=-\frac{(d-1)(d-3)}{\left(1+|x|^{2}\right)^{3 / 2}}-\frac{6(d-3)}{\left(1+|x|^{2}\right)^{5 / 2}}-\frac{15}{\left(1+|x|^{2}\right)^{7 / 2}}-\frac{(d-1)(d-3)}{|x|^{3}}, \\
-\Delta^{2} \Phi(x) \geq \frac{d(d+2)}{8 \sqrt{2}} \chi_{B(0,1)}(x)+\frac{(d-1)(d-3)}{|x|^{3}} \\
1 \leq \Phi^{\prime}(r)=\frac{r}{\left(1+r^{2}\right)^{1 / 2}}+1 \leq 2 \quad r \geq 0, \\
0 \leq r \Phi^{\prime \prime}(r)=\frac{r}{\left(1+r^{2}\right)^{1 / 2}}-\frac{r^{3}}{\left(1+r^{2}\right)^{3 / 2}} \leq 1 \quad r \geq 0 \\
\frac{1}{2 \sqrt{2}} \leq \inf \left\{\Phi^{\prime \prime}(r), \frac{\Phi^{\prime}(r)-1}{r}\right\} \quad r \leq 1
\end{gathered}
$$

Now we define for $R>0$

$$
\Phi_{R}(x)=R \Phi\left(\frac{x}{R}\right) .
$$

As a consequence of (2) and (28):

$$
\begin{gathered}
-\frac{1}{4} \Delta^{2} \Phi_{R}(x)-\frac{1}{2} \nabla \Phi_{R}(x) \cdot \nabla V(x) \\
\geq \frac{\gamma}{2} \frac{V(x)}{|x|}+\frac{d(d+2)}{32 \sqrt{2} R^{3}} \chi_{B(0, R)}(x)+\frac{\eta}{4} \frac{(d-1)(d-3)}{|x|^{3}} .
\end{gathered}
$$


By using (28)-(31) and (25), we obtain, by inserting all the estimates in (108), that

$$
\begin{gathered}
\frac{1}{R} \int_{B(0, R)}|\nabla u(x)|^{2} d x+\int_{\mathbb{R}^{d}} \frac{V(x)}{|x|}|u(x)|^{2} d x \\
+(d-3) \int_{\mathbb{R}^{d}} \frac{|u(x)|^{2}}{|x|^{3}} d x+\frac{1}{R^{3}} \int_{B(0, R)}|u(x)|^{2} d x \\
\leq C \int_{\mathbb{R}^{d}}|f(x)||\nabla u(x)| d x+C \int_{\mathbb{R}^{d}}|f(x)| \frac{|u(x)|}{|x|} d x+C|\epsilon| \int_{\mathbb{R}^{d}}|u(x)||\nabla u(x)| d x .
\end{gathered}
$$

To finish we need the terms on the left hand side of (33) to be finite but, before seeing this, let us deal with the case $d=3$. In this case, we can find, for $R>0$, a radial function $\Phi$ (see Lemma 6.1 in Appendix 2) such that:

- $\Delta^{2} \Phi(x)=-\frac{c_{1}}{R^{3}} \chi_{(0, R)}(x)-\frac{W(|x|)}{|x|}, x \in \mathbb{R}^{3}$, where $W$ is the function in (3).

- $\inf _{r>0}\left\{\Phi^{\prime}(r), \Phi^{\prime \prime}(r)\right\} \geq 0$.

- $\inf _{r \in(0, R)}\left\{\frac{\Phi^{\prime}(r)}{r}, \Phi^{\prime \prime}(r)\right\} \geq \frac{c_{2}}{R}$.

- $c_{3}<\Phi^{\prime}(r)<\kappa<\frac{1}{2}, \quad r>0$.

for some $c_{1}, c_{2}, c_{3}$ and $\kappa$ positive constants.

If we use the above inequalities and (3), we have

$$
\begin{gathered}
-\frac{1}{4} \Delta^{2} \Phi(x)-\frac{1}{2} \nabla \Phi(x) \cdot V(x)=\frac{c_{1}}{4 R^{3}} \chi_{(0, R)}(x)+\frac{W(|x|)}{4|x|}-\frac{1}{2} \Phi^{\prime}(x) \partial_{r} V(x) \\
\geq \frac{c_{1}}{4 R^{3}} \chi_{(0, R)}(x)+\frac{\gamma V(x)}{2|x|}+\frac{w(|x|)}{2|x|}\left(\frac{1}{2}-\Phi^{\prime}(|x|)\right) \\
\geq \frac{c_{1}}{4 R^{3}} \chi_{(0, R)}(x)+\frac{\gamma V(x)}{2|x|}+\frac{w(|x|)}{2|x|}\left(\frac{1}{2}-\kappa\right) .
\end{gathered}
$$

Now, since $\frac{1}{2}-\kappa>0$, we can use (108) as in the case $d>3$ to conclude that for $d \geq 3$ we have

$$
\begin{gathered}
\frac{1}{R} \int_{B(0, R)}|\nabla u(x)|^{2} d x+\int_{\mathbb{R}^{d}} \frac{V(x)}{|x|}|u(x)|^{2} d x+(d-3) \int_{\mathbb{R}^{d}} \frac{|u(x)|^{2}}{|x|^{3}} d x \\
+\chi(d) \int_{\mathbb{R}^{3}} \frac{W(|x|)}{|x|}|u(x)|^{2} d x+\frac{1}{R^{3}} \int_{B(0, R)}|u(x)|^{2} d x \\
\leq C \int_{\mathbb{R}^{d}}|f(x)||\nabla u(x)| d x+C \int_{\mathbb{R}^{d}}|f(x)| \frac{|u(x)|}{|x|} d x+C|\epsilon| \int_{\mathbb{R}^{d}}|u(x)||\nabla u(x)| d x .
\end{gathered}
$$

We check now that the terms on the left hand side of (22) are finite. Notice that, since the right hand side of (35) does not depend on $R>0$, it suffices to check that it is finite.

On one hand the classical theory guarantees, with our a priori condition $f \in L^{2}$, the existence and uniqueness of solution of (17) in $L^{2}\left(\mathbb{R}^{d}\right)$. On the other hand, by 
taking $\varphi=1$ in (106), since $V \geq 0$, we obtain

$$
\int|\nabla u|^{2} d x \leq(\max \{0, \tau\}+1)\|u\|_{2}^{2}+\|f\|_{2}^{2},
$$

and hence we obtain that $u \in W^{1,2}$. Therefore,

$$
\begin{aligned}
& \int_{\mathbb{R}^{n}}|f(x)||\nabla u(x)| d x \leq\|f\|_{L^{2}}\|\nabla u\|_{L^{2}}<\infty, \\
& \int_{\mathbb{R}^{n}}|u(x)||\nabla u(x)| d x \leq\|u\|_{L^{2}}\|\nabla u\|_{L^{2}}<\infty,
\end{aligned}
$$

and using Hardy's inequality, we have

$$
\int_{\mathbb{R}^{n}}|f(x)| \frac{|u(x)|}{|x|} d x \leq C \int_{\mathbb{R}^{d}}|f(x)|^{2} d x+C \int_{\mathbb{R}^{d}}|\nabla u(x)|^{2} d x<\infty .
$$

Finally, by taking sup in (35) we get

$$
\begin{aligned}
& \left\|\left.\left|\nabla u \|_{X}^{2}+\int_{\mathbb{R}^{d}} \frac{V(x)}{|x|}\right| u(x)\right|^{2} d x+\chi(d) \int_{\mathbb{R}^{3}} \frac{W(|x|)}{|x|}|u(x)|^{2} d x\right. \\
& +\sup _{R>0} \frac{1}{R^{3}} \int_{B(0, R)}|u(x)|^{2} d x+(d-3) \int_{\mathbb{R}^{d}} \frac{|u(x)|^{2}}{|x|^{3}} d x<\infty \quad d \geq 3,
\end{aligned}
$$

as desired. Now, to obtain the a priori estimate, we bound the terms on the right hand side of (35).

We start by writing

$$
\begin{aligned}
& C \int_{\mathbb{R}^{d}}|f(x)| \frac{|u(x)|}{|x|} d x \leq C \sum_{j \in \mathbb{Z}} \int_{C(j)}|f(x)| \frac{|u(x)|}{|x|} d x \\
& \leq C \sum_{j \in \mathbb{Z}}\left(2^{j} \int_{C(j)}|f(x)|^{2} d x\right)^{1 / 2}\left(\frac{1}{2^{3 j}} \int_{C(j)}|u(x)|^{2} d x\right)^{1 / 2} \\
& \leq C\|f\|_{X^{*}}\left(\sup _{R>0} \frac{1}{R^{3}} \int_{B(0, R)}|u(x)|^{2} d x\right)^{1 / 2} \\
& \leq C\|f\|_{X^{*}}^{2}+\frac{1}{2} \sup _{R>0} \frac{1}{R^{3}} \int_{B(0, R)}|u(x)|^{2} d x .
\end{aligned}
$$

We also have the pairing

$$
C \int_{\mathbb{R}^{d}}\left|f(x)\|\nabla u(x) \mid d x \leq C\| f\left\|_{X^{*}}\right\| \nabla u\left\|_{X} \leq C\right\| f\left\|_{X^{*}}^{2}+\frac{1}{2}\right\| \nabla u \|_{X}^{2} .\right.
$$

To treat the third term on the right hand side of (35), we take $\varphi=1$ in (107) to obtain

$$
|\epsilon| \int_{\mathbb{R}^{d}}|u(x)|^{2} d x \leq \int_{\mathbb{R}^{d}}|f(x)||u(x)| d x .
$$

and if we again take $\varphi=1$ in (106) and by using (43), we get

$$
\begin{gathered}
|\epsilon| \int_{\mathbb{R}^{d}}|\nabla u(x)|^{2} d x \leq \max \{0, \tau\}|\epsilon| \int_{\mathbb{R}^{d}}|u(x)|^{2} d x+|\epsilon| \int_{\mathbb{R}^{d}}|f(x)||u(x)| d x \\
\leq(\max \{0, \tau\}+|\epsilon|) \int_{\mathbb{R}^{d}}|f(x)||u(x)| d x .
\end{gathered}
$$


Now we use Cauchy-Schwartz inequality, (43), (44) to have

$$
C|\epsilon| \int_{\mathbb{R}^{d}}|u(x)||\nabla u(x)| d x \leq C\|f\|_{X^{*}}^{2}+\frac{1}{4}(|\epsilon|+\max \{0, \tau\})\|u\|_{X}^{2} .
$$

From this, (35), (41) and by taking sup in $R>0$, we have

$$
\begin{aligned}
& \|\nabla u\|_{X}^{2}+\int_{\mathbb{R}^{d}} \frac{V(x)}{|x|}|u(x)|^{2} d x+\sup _{R>0} \frac{1}{R^{3}} \int_{B(0, R)}|u(x)|^{2} d x \\
& +(d-3) \int_{\mathbb{R}^{d}} \frac{|u(x)|^{2}}{|x|^{3}} d x+\chi(d) \int_{\mathbb{R}^{3}} \frac{W(|x|)}{|x|}|u(x)|^{2} d x \leq C\|f\|_{X^{*}} \\
& +\frac{1}{2} \sup _{R>0} \frac{1}{R^{3}} \int_{B(0, R)}|u(x)|^{2} d x+\frac{1}{2}\|\nabla u\|_{X}^{2}+\frac{1}{4}(|\epsilon|+\max \{0, \tau\})\|u\|_{X}^{2} .
\end{aligned}
$$

and we obtain (22).

Proof of of estimate (23).

For $R>0$ fixed, we consider the function

$$
\varphi_{R}(x)=\frac{1}{R} \chi_{\{|x|<R\}}(x)+\left(\frac{2}{R}-\frac{|x|}{R^{2}}\right) \chi_{\{R \leq|x|<2 R\}} .
$$

We have that

and

$$
\frac{1}{R} \chi_{\{|x|<R\}}(x) \leq \varphi_{R}(x) \leq \frac{1}{R} \chi_{\{|x|<2 R\}}(x)
$$

$$
\left|\nabla \varphi_{R}(x)\right| \leq \frac{1}{R^{2}} \chi_{\{R<|x|<2 R\}}(x) .
$$

If we take in (107) $\varphi=\varphi_{R}$, using the previous estimates we obtain that

$$
\begin{gathered}
\frac{|\epsilon|}{R} \int_{B(0, R)}|u(x)|^{2} d x \leq \frac{1}{R^{2}} \int_{R<|x|<2 R}|\nabla u(x)||u(x)| d x \\
+\frac{1}{R} \int_{B(0,2 R)}|f(x)||u(x)| d x .
\end{gathered}
$$

Using Cauchy-Schwartz inequality, we have

$$
\frac{1}{R^{2}} \int_{R<|x|<2 R}\left|\nabla u ( x ) \left\|\left.u(x)\left|d x \leq \frac{1}{4}\|\nabla u\|_{X}^{2}+\frac{1}{4} \sup _{R>0} \frac{1}{R^{3}} \int_{B(0, R)}\right| u(x)\right|^{2} d x .\right.\right.
$$

Finally, as in (41)

$$
\begin{aligned}
& \frac{1}{R} \int_{B(0,2 R)}|f(x)||u(x)| d x \leq 2 \int_{\mathbb{R}^{d}}|f(x)| \frac{|u(x)|}{|x|} d x \\
& \quad \leq \frac{1}{2}\|f\|_{X^{*}}^{2}+\frac{1}{2} \sup _{R>0} \frac{1}{R^{3}} \int_{B(0, R)}|u(x)|^{2} d x .
\end{aligned}
$$

The result follows from (47), (48) and (49).

Proof of of estimate (24).

Following [13], for $R>0$ fixed, we consider two functions $\Phi_{R}$ and $\varphi_{R}$ given by

$$
\nabla \Phi_{R}(x)=\frac{x}{R} \chi_{\{|x|<R\}}(x)+\frac{x}{|x|} \chi_{\{|x| \geq R\}}(x),
$$




$$
\varphi_{R}(x)=\frac{1}{2 R} \chi_{\{|x|<R\}}(x) .
$$

Some calculations give us the following identities which hold in the distributional sense:

$$
\begin{gathered}
\Delta \Phi_{R}(x)=\frac{d}{R} \chi_{\{|x|<R\}}(x)+\frac{d-1}{|x|} \chi_{\{|x|>R\}}(x), \\
D_{i j}^{2} \Phi_{R}(x)=\frac{\delta_{i j}}{R} \chi_{\{|x|<R\}}(x)+\left(\frac{\delta_{i j}}{|x|}-\frac{x_{i} x_{j}}{|x|^{3}}\right) \chi_{\{|x|>R\}}(x),
\end{gathered}
$$

and

$$
\Delta\left(2 \varphi_{R}-\Delta \Phi_{R}\right)(x)=\frac{(d-1)}{R^{2}} \delta_{\{|x|=R\}}+\frac{(d-1)(d-3)}{|x|^{3}} \chi_{\{|x|>R\}}(x),
$$

where

$$
\delta_{i j}=\left\{\begin{array}{lll}
1 & \text { if } \quad i=j \\
0 & \text { if } \quad i \neq j
\end{array}\right.
$$

It is easy to check that

$$
\begin{gathered}
\nabla \bar{u}(x) \cdot D^{2} \Phi_{R}(x) \cdot \nabla u(x) \geq \frac{|\nabla u(x)|^{2}}{R} \chi_{\{|x|<R\}}(x), \\
\left|\varphi_{R}\right| \leq \frac{1}{2|x|}, \quad\left|\nabla \Phi_{R}\right| \leq 1, \quad\left|\Delta \Phi_{R}\right| \leq \frac{d}{|x|} .
\end{gathered}
$$

From (40) we know that

$$
(d-3) \int_{\mathbb{R}^{d}} \frac{|u(x)|^{2}}{|x|^{3}} d x+\chi(d) \int_{\mathbb{R}^{3}} \frac{W(|x|)}{|x|}|u(x)|^{2} d x<\infty .
$$

Then using (2) and (3),

$$
\begin{aligned}
& -\frac{1}{2} \int_{\mathbb{R}^{d}} \nabla V(x) \cdot \nabla \Phi_{R}(x)|u(x)|^{2} d x \geq \frac{\gamma}{2 R} \int_{B(0, R)} V(x)|u(x)|^{2} d x \\
& +\frac{\gamma}{2} \int_{|x|>R} \frac{V(x)}{|x|}|u(x)|^{2} d x-(1-\eta) \frac{(d-1)(d-3)}{4} \int_{\mathbb{R}^{d}} \frac{|u(x)|^{2}}{|x|^{3}} d x \quad d>3
\end{aligned}
$$

and

$$
\begin{aligned}
& -\frac{1}{2} \int_{\mathbb{R}^{3}} \nabla V(x) \cdot \nabla \Phi_{R}(x)|u(x)|^{2} d x \geq \frac{\gamma}{2 R} \int_{B(0, R)} V(x)|u(x)|^{2} d x \\
& +\frac{\gamma}{2} \int_{|x|>R} \frac{V(x)}{|x|}|u(x)|^{2} d x-\frac{1}{2} \int_{\mathbb{R}^{3}} \frac{W(|x|)}{|x|}|u(x)|^{2} d x .
\end{aligned}
$$

If $d \geq 3$, with the above inequalities, by using $\Phi=\Phi_{R}$ and $\varphi=\varphi_{R}$ in (109) and that $\tau \geq 0$, we have that

$$
\begin{aligned}
& \frac{1}{2 R} \int_{B(0, R)}|\nabla u(x)|^{2} d x+\frac{(d-1)}{4 R^{2}} \int_{|x|=R}|u(x)|^{2} d \sigma_{R}(x)+\frac{\tau}{2 R} \int_{B(0, R)}|u(x)|^{2} d x \\
& +\frac{d-3}{4} \int_{|x|>R} \frac{|u(x)|^{2}}{|x|^{3}} d x+\frac{\gamma}{2 R} \int_{B(0, R)} V|u(x)|^{2} d x+\frac{\gamma}{2} \int_{|x|>R} \frac{V(x)}{|x|}|u(x)|^{2} d x \\
& -(1-\eta) \frac{d-3}{4} \int_{\mathbb{R}^{d}} \frac{|u(x)|^{2}}{|x|^{3}} d x-\frac{1}{2} \chi(d) \int_{\mathbb{R}^{3}} \frac{W(|x|)}{|x|}|u(x)|^{2} d x \leq \int_{\mathbb{R}^{d}}|f||\nabla u(x)| d x \\
& +\frac{(d+1)}{2} \int_{\mathbb{R}^{d}}|f(x)| \frac{|u(x)|}{|x|} d x+|\epsilon| \int_{\mathbb{R}^{d}}|\nabla u(x)||u(x)| d x+\frac{1}{2} \int_{\mathbb{R}^{d}} \frac{V(x)}{|x|}|u(x)|^{2} d x
\end{aligned}
$$


If we use (36)-(39) and (40), we have

$$
\begin{gathered}
\|\nabla u\|_{X}^{2}+\int_{\mathbb{R}^{d}} \frac{V(x)}{|x|}|u(x)|^{2} d x+\sup _{R>0} \frac{1}{R^{2}} \int_{|x|=R}|u(x)|^{2} d \sigma_{R}(x) \\
+\left\|V^{\frac{1}{2}} u\right\|_{X}^{2}+\tau\|u\|_{X}^{2}<\infty .
\end{gathered}
$$

Let $\delta>0$ be such that

$$
\int_{0}^{\infty} t W(t) d t<\frac{1}{2}-\delta
$$

use (41), (42), (45), (22) and take sup in (54), then we have

$$
\begin{aligned}
& \|\nabla u\|_{X}^{2}+\sup _{R>0} \frac{1}{R^{2}} \int_{|x|=R}|u(x)|^{2} d \sigma_{R}(x)+\tau\|u\|_{X}^{2}+\left\|V^{\frac{1}{2}} u\right\|_{X}^{2} \\
& +\int_{\mathbb{R}^{d}} \frac{V(x)}{|x|}|u(x)|^{2} d x+(d-3) \int_{\mathbb{R}^{d}} \frac{|u(x)|^{2}}{|x|^{3}} d x-\frac{1}{2} \chi(d) \int_{\mathbb{R}^{3}} \frac{W(|x|)}{|x|}|u(x)|^{2} d x \\
& \leq C\|f\|_{X^{*}}^{2}+\frac{1}{2} \sup _{R>0} \frac{1}{R^{3}} \int_{B(0, R)}|u(x)|^{2} d x+\frac{1}{2}\|\nabla u\|_{X}^{2}+\frac{1}{2}(|\epsilon|+\tau)\|u\|_{X}^{2} .
\end{aligned}
$$

Estimate (24) follows from this and by using

$$
\sup _{R>0} \frac{1}{R^{3}} \int_{B(0, R)}|u(x)|^{2} d x \leq \sup _{R>0} \frac{1}{R^{2}} \int_{|x|=R}|u(x)|^{2} d \sigma_{R}(x),
$$

and

$$
\begin{aligned}
& \left(\frac{1}{2}+\delta\right) \int_{\mathbb{R}^{3}} \frac{W(|x|)}{|x|}|u(x)|^{2} d x=\left(\frac{1}{2}+\delta\right) \int_{0}^{\infty} \frac{W(t)}{t} \int_{|x|=t}|u(x)|^{2} d \sigma_{t}(x) d t \\
& <\left(\frac{1}{4}-\delta^{2}\right) \sup _{R>0} \frac{1}{R^{2}} \int_{|x|=R}|u(x)|^{2} d \sigma_{R}(x) .
\end{aligned}
$$

2.2. Proof of the theorem 1.1. We start with the proof of the estimate (5).

For simplicity, for $R>0$ fixed, we introduce the function $\psi_{R}$ defined by

$$
\psi_{R}(x)=\frac{1}{R\left(1+\frac{|x|^{2}}{R^{2}}\right)} .
$$

Since $\psi_{R}(x)>\frac{1}{2 R} \chi_{B(0, R)}(x)$, to show that (5) holds, it is enough to prove that for all $R>0$, there exists a positive constant $C$ independent of $R$, such that

$$
\int_{-\infty}^{\infty} \int_{\mathbb{R}^{d}} \psi_{R}(x)\left|D^{\frac{1}{2}} e^{i t H} u_{0}(x)\right|^{2} d x d t \leq C\left\|u_{0}\right\|_{2}^{2}
$$

This estimate is equivalent to say that the operator $\psi_{R}^{\frac{1}{2}} D^{\frac{1}{2}}$ is $H$-smooth (see [15], v.IV or [10]). We will prove, further, that $\psi_{R}^{\frac{1}{2}} D^{\frac{1}{2}}$ is $H$-supersmooth, which means, (see [10]), that for all $f \in \mathcal{D}\left(D^{\frac{1}{2}} \psi_{R}^{\frac{1}{2}}\right) \subset L^{2}\left(\mathbb{R}^{d}\right), \tau \in \mathbb{R}$ and $\epsilon>0$, there exists a positive constant $C$ independent of $\tau$ and $\epsilon$ such that

$$
\left\|\psi_{R}^{\frac{1}{2}} D^{\frac{1}{2}} R_{H}(\tau \pm i \epsilon) D^{\frac{1}{2}} \psi_{R}^{\frac{1}{2}} f\right\|_{L^{2}} \leq C\|f\|_{L^{2}} .
$$

In our case, the constant $C$ also has to be independent of $R$.

This estimate can be obtained, using complex interpolation of operators, from the following estimate and its dual version:

$$
\left\|\psi_{R}^{\frac{1}{2}} D^{1+i \eta} R_{H}(\tau \pm i \epsilon) D^{-i \eta} \psi_{R}^{\frac{1}{2}} f\right\|_{L^{2}} \leq C\|f\|_{L^{2}}, \quad \forall \eta \in \mathbb{R} .
$$


This inequality can be written as

$$
\left\|D^{1+i \eta} R_{H}(\tau \pm i \epsilon) D^{-i \eta} f\right\|_{L^{2}\left(\psi_{R}\right)} \leq C\|f\|_{L^{2}\left(\psi_{R}^{-1}\right)} .
$$

We see that for any $\gamma \in \mathbb{R}$,

$$
\begin{aligned}
\left\|D^{i \gamma} f\right\|_{L^{2}\left(\psi_{R}\right)} & \leq C\|f\|_{L^{2}\left(\psi_{R}\right)}, \\
\left\|D^{-i \gamma} f\right\|_{L^{2}\left(\psi_{R}^{-1}\right)} & \leq C\|f\|_{L^{2}\left(\psi_{R}^{-1}\right)}, \\
\|D f\|_{L^{2}\left(\psi_{R}\right)} & \leq C\|\nabla f\|_{L^{2}\left(\psi_{R}\right)},
\end{aligned}
$$

with $C$ a positive constant independent of $R$. Therefore, to show that (60) holds, it will be enough to prove that

$$
\left\|\nabla R_{H}(\tau \pm i \epsilon) f\right\|_{L^{2}\left(\psi_{R}\right)} \leq C\|f\|_{L^{2}\left(\psi_{R}^{-1}\right)} .
$$

Estimates (61), (62) and (63) are consequence of the fact that $\psi_{R}$ and $\psi_{R}^{-1}$ are weights in the class $A_{2}$ (see [5]). This means that for any cube $Q$ in $\mathbb{R}^{d}$, there exists a constant $C$ independent of $Q$ such that

$$
\left(\frac{1}{|Q|} \int_{Q} \psi_{R}(x) d x\right)\left(\frac{1}{|Q|} \int_{Q} \frac{1}{\psi_{R}(x)} d x\right) \leq C .
$$

One can check that the constant $C$ above is independent of $R$.

Now, since $\psi_{R}(x) \leq 1 / R$ and $\psi_{R}(x) \leq R /|x|^{2}$, we have that

$$
\begin{aligned}
\left\|\nabla R_{H}(\tau \pm i \epsilon) f\right\|_{L^{2}\left(\psi_{R}\right)}^{2} \leq & \frac{1}{R} \int_{B(0, R)}\left|\nabla R_{H}(\tau \pm i \epsilon) f(x)\right|^{2} d x \\
& +R \int_{|x| \geq R} \frac{\left|\nabla R_{H}(\tau \pm i \epsilon) f(x)\right|^{2}}{|x|^{2}} d x .
\end{aligned}
$$

We control the second term in the previous inequality by taking $k \in \mathbb{N}$ such that

$$
2^{k}<R \leq 2^{k+1}
$$

Thus

$$
\begin{gathered}
R \int_{|x| \geq R} \frac{\left|\nabla R_{H}(\tau \pm i \epsilon) f(x)\right|^{2}}{|x|^{2}} d x \leq R \sum_{j \geq k}^{\infty} \int_{C_{j}} \frac{\left|\nabla R_{H}(\tau \pm i \epsilon) f(x)\right|^{2}}{|x|^{2}} d x \\
\leq R\left\|\nabla R_{H}(\tau \pm i \epsilon) f\right\|_{X}^{2} \sum_{j \geq k}^{\infty} \frac{1}{2^{j}} \leq C\left\|\nabla R_{H}(\tau \pm i \epsilon) f\right\|_{X}^{2} .
\end{gathered}
$$

Inserting this in (65) we have that

$$
\left\|\nabla R_{H}(\tau \pm i \epsilon) f\right\|_{L^{2}\left(\psi_{R}\right)}^{2} \leq C\left\|\nabla R_{H}(\tau \pm i \epsilon) f\right\|_{X}^{2} .
$$

Furthermore, taking into account that $\psi_{R}^{-1}(x) \geq R, \psi_{R}^{-1}(x) \geq|x|^{2} / R$, and for $k \in \mathbb{N}$ satifying (66), we have that

$$
\|f\|_{X^{*}}^{2} \leq R \int_{B(0, R)}|f(x)|^{2} d x+\frac{1}{R} \int_{|x| \geq R}|f(x)|^{2}|x|^{2} d x \leq C\|f\|_{L^{2}\left(\psi_{R}^{-1}\right)}^{2} .
$$

After (67), (20) and the above inequality we get (64), from which (5) follows. 
Arguing in a similar way to show that (6) holds, we will prove that the operator of multiplication by the function $\psi_{R}^{\frac{1}{2}} V^{\frac{1}{4}}$ is $H$-supersmooth. We prove then that for all $f \in \mathcal{D}\left(\psi_{R}^{\frac{1}{2}} V^{\frac{1}{4}}\right) \subset L^{2}\left(\mathbb{R}^{d}\right), \tau \in \mathbb{R}$ and $\epsilon>0$, there exists a positive constant $C$ independent of $\tau, \epsilon$ and $R$ such that

$$
\left\|\psi_{R}^{\frac{1}{2}} V^{\frac{1}{4}} R_{H}(\tau \pm i \epsilon) \psi_{R}^{\frac{1}{2}} V^{\frac{1}{4}} f\right\|_{L^{2}} \leq C\|f\|_{L^{2}} .
$$

Again this estimate can be obtained, using complex interpolation of operators, from the following estimate and its dual:

$$
\left\|\psi_{R}^{\frac{1}{2}} V^{\frac{1+i \gamma}{2}} R_{H}(\tau \pm i \epsilon) V^{-i \frac{\gamma}{2}} \psi_{R}^{\frac{1}{2}} f\right\|_{L^{2}} \leq C\|f\|_{L^{2}}, \quad \forall \gamma \in \mathbb{R} .
$$

This inequality can be written as

$$
\left\|V^{\frac{1+i \gamma}{2}} R_{H}(\tau \pm i \epsilon) V^{-i \frac{\gamma}{2}} f\right\|_{L^{2}\left(\psi_{R}\right)} \leq C\|f\|_{L^{2}\left(\psi_{R}^{-1}\right)} .
$$

Since

$$
\begin{aligned}
\left\|V^{i \gamma} f\right\|_{L^{2}\left(\psi_{R}\right)} & \leq C\|f\|_{L^{2}\left(\psi_{R}\right)}, \\
\left\|V^{-i \gamma} f\right\|_{L^{2}\left(\psi_{R}^{-1}\right)} & \leq C\|f\|_{L^{2}\left(\psi_{R}^{-1}\right)},
\end{aligned}
$$

with $C$ a positive constant independent of $R$, to show (69) we can reduce to prove that

$$
\left\|V^{\frac{1}{2}} R_{H}(\tau \pm i \epsilon) f\right\|_{L^{2}\left(\psi_{R}\right)} \leq C\|f\|_{L^{2}\left(\psi_{R}^{-1}\right)} .
$$

Finally, since $\psi_{R}(x) \leq 1 /|x|$, from (21) and (68), we have that

$$
\begin{gathered}
\left\|V^{\frac{1}{2}} R_{H}(\tau \pm i \epsilon) f\right\|_{L^{2}\left(\psi_{R}\right)} \leq C\left\|R_{H}(\tau \pm i \epsilon) f\right\|_{L^{2}\left(\frac{V(x)}{|x|}\right)} \\
\leq C\|f\|_{X^{*}} \leq C\|f\|_{L^{2}\left(\psi_{R}^{-1}\right)}
\end{gathered}
$$

Remark 2.3. Estimates (22) and (23) hold for $\gamma=0$, nevertheless our proof of estimate (24) does not work in this case. To obtain (18) we need also (24). This is the reason for which theorem 1.1 is not a generalization of the work 3 .

\section{Some attractive Perturbations}

3.1. Estimates for the resolvent. In this section, we give a priori estimate for the resolvent of the operator $H$ defined in the Theorem 1.2 that will be the key to prove this theorem.

Theorem 3.1. Let $V$ and $n$ be two real valued functions satisfying the conditions of Theorem 1.2, and let $u$ be a solution of the equation

$$
-\Delta u+(V(x)+n(x)) u \pm i \epsilon u-\tau u=f, \quad \epsilon \neq 0, x \in \mathbb{R}^{d} \quad d \geq 3 .
$$


Then, given $\rho>0$ and $\tau_{0}>0$, the following estimates hold:

$$
\begin{gathered}
+\left\||n|^{\frac{1}{2}} u\right\|_{X_{\rho}}^{2}+(d-3) \int_{\mathbb{R}^{d}} \frac{|u(x)|^{2}}{|x|^{3}} d x+\tau\|u\|_{X_{\rho}}^{2}+\int_{\mathbb{R}^{d}} \frac{V(x)}{|x|}|u(x)|^{2} d x \\
+\chi(d) \int_{\mathbb{R}^{3}} \frac{W(|x|)}{|x|}|u(x)|^{2} d x \leq C\left(\beta_{\rho}\right)\left(\|f\|_{X_{\rho}^{*}}^{2}+\left\|\frac{f}{|n|^{\frac{1}{2}}}\right\|_{X_{\rho}^{*}}^{2}\right), \quad \forall \tau \geq 0 . \\
\|\nabla u\|_{X_{\rho}}^{2}+\sup _{R \geq \rho} \frac{1}{R^{2}} \int_{|x|=R}|u(x)|^{2} d \sigma_{R}(x) \\
+\left\||n|^{\frac{1}{2}} u\right\|_{X_{\rho}}^{2}+(d-3) \int_{\mathbb{R}^{d}} \frac{|u(x)|^{2}}{|x|^{3}} d x+\tau\|u\|_{X_{\rho}}^{2}+\int_{\mathbb{R}^{d}} \frac{V(x)}{|x|}|u(x)|^{2} d x \\
+\chi(d) \int_{\mathbb{R}^{3}} \frac{W(|x|)}{|x|}|u(x)|^{2} d x \leq C\left(\beta_{\rho}, \tau_{0}\right)\|f\|_{X_{\rho}^{*}}^{2}, \quad \forall \tau \geq \tau_{0} .
\end{gathered}
$$

where $\chi(d)$ is the function defined in (19), $W$ the function in (3), $C\left(\beta_{\rho}\right)$ and $C\left(\beta_{\rho}, \tau_{0}\right)$ are constants independent of $\epsilon$ and $\tau$.

Remark 3.2. Estimate (72) says that

$$
\left\|\nabla R_{H}(\tau \pm i \epsilon) f\right\|_{X_{\rho}} \leq C\|f\|_{X_{\rho}^{*}},
$$

with $C$ independent of $\epsilon \neq 0$ and $\tau \geq 0$ and

$$
\tau^{1 / 2}\left\|R_{H}(\tau \pm i \epsilon) f\right\|_{X_{\rho}} \leq C\|f\|_{X_{\rho}^{*}},
$$

with $C$ independent of $\epsilon \neq 0$ and $\tau \geq \tau_{0}$.

Proof. We follow the scheme of the proof of Theorem 2.1. Let $u$ denote a solution of (70) when $f \in L^{2}\left(\mathbb{R}^{d}\right)$.

We start with the proof of (71). This will be a consequence of

$$
\left\{\begin{array}{l}
\|\nabla u\|_{X_{\rho}}^{2}+\sup _{R \geq \rho} \frac{1}{R^{2}} \int_{|x|=R}|u(x)|^{2} d \sigma_{R}(x)+\int_{\mathbb{R}^{d}} \frac{V(x)}{|x|}|u(x)|^{2} d x \\
+\left\||n|^{\frac{1}{2}} u\right\|_{X_{\rho}}^{2}+(d-3) \int_{\mathbb{R}^{d}} \frac{|u(x)|^{2}}{|x|^{3}} d x+\chi(d) \int_{\mathbb{R}^{3}} \frac{W(|x|)}{|x|}|u(x)|^{2} d x \\
+\tau\|u\|_{X_{\rho}}^{2} \leq C\left(\|f\|_{X_{\rho}^{*}}^{2}+\left\|\frac{f}{|n|^{\frac{1}{2}}}\right\|_{X_{\rho}^{*}}^{2}\right)+\delta|\epsilon|\|u\|_{X_{\rho}}^{2}
\end{array}\right.
$$

and (23), that is true for the solutions of (170).

We begin considering the case $d=3$. By (3) and Lemma 6.1 in Appendix 2, we can choose three positive constants $\alpha, \epsilon$ and $\delta$ an a radial function $\Phi_{R}$ such that

- $\alpha+\frac{\epsilon}{6}+\int_{0}^{\infty} t\left(W(t)+\frac{\delta}{t} \chi_{(0, \rho)}(t)\right) d t<\frac{1}{2}$.

- $\Delta \Phi_{R}(x)=-\frac{\epsilon}{R^{3}} \chi_{(0, R)}(|x|)-\frac{W(|x|)}{|x|}-\frac{\delta}{|x|^{2}} \chi_{(0, \rho)}(|x|)$.

- $\inf _{r>0}\left\{\Phi^{\prime}(r), \Phi^{\prime \prime}(r)\right\} \geq 0$.

- $\inf _{r \in(0, R)}\left\{\frac{\Phi^{\prime}(r)}{r}, \Phi^{\prime \prime}(r)\right\} \geq \frac{C \epsilon}{R}$.

- $\alpha<\Phi^{\prime}(r)<\kappa<\frac{1}{2}, \quad r>0$. 
Inserting this function in (108) and following the proof of (22), we can check that

$$
\begin{gathered}
\quad \frac{C_{1}}{R} \int_{B(0, R)}|\nabla u(x)|^{2} d x+\frac{C_{1}}{R^{3}} \int_{B(0, R)}|u(x)|^{2} d x+C_{1} \int_{B(0, \rho)} \frac{|u(x)|^{2}}{|x|^{2}} d x \\
+C_{1} \int_{\mathbb{R}^{3}} \frac{W(|x|)}{|x|}|u(x)|^{2} d x+\alpha \frac{\gamma}{2} \int_{\mathbb{R}^{3}} \frac{V(x)}{|x|}|u(x)|^{2} d x \leq C \int_{\mathbb{R}^{3}}|f(x)||\nabla u(x)| d x \\
+C \int_{\mathbb{R}^{3}}|f(x)| \frac{|u(x)|}{|x|} d x+|\epsilon| \int_{\mathbb{R}^{3}}|u(x)||\nabla u(x)| d x+\frac{1}{4} \int_{\mathbb{R}^{3}} \frac{\nabla n(x) \cdot x}{|x|}|u(x)|^{2} d x,
\end{gathered}
$$

with $C$ and $C_{1}$ absolute constants.

In a similar way we have also the analogous for $d>3$, see specially (29),

$$
\begin{gathered}
\frac{C_{1}}{R} \int_{B(0, R)}|\nabla u(x)|^{2} d x+\frac{C_{1}}{R^{3}} \int_{B(0, R)}|u(x)|^{2} d x+C_{1}(d-3) \int_{\mathbb{R}^{d}} \frac{|u(x)|^{2}}{|x|^{3}} d x \\
+\frac{\gamma}{2} \int_{\mathbb{R}^{d}} \frac{V(x)}{|x|}|u(x)|^{2} d x \leq C \int_{\mathbb{R}^{d}}|f(x)||\nabla u(x)| d x+C \int_{\mathbb{R}^{d}}|f(x)| \frac{|u(x)|}{|x|} d x \\
+|\epsilon| \int_{\mathbb{R}^{d}}|u(x)||\nabla u(x)| d x+\int_{\mathbb{R}^{d}} \frac{\nabla n(x) \cdot x}{|x|}|u(x)|^{2} d x .
\end{gathered}
$$

Both together write as

$$
\begin{aligned}
& \frac{C_{1}}{R} \int_{B(0, R)}|\nabla u(x)|^{2} d x+\frac{C_{1}}{R^{3}} \int_{B(0, R)}|u(x)|^{2} d x+C_{1}(d-3) \int_{\mathbb{R}^{d}} \frac{|u(x)|^{2}}{|x|^{3}} d x \\
& +(\alpha \chi(d)+(1-\chi(d))) \frac{\gamma}{2} \int_{\mathbb{R}^{d}} \frac{V(x)}{|x|}|u(x)|^{2} d x+C_{1} \chi(d) \int_{B(0, \rho)} \frac{|u(x)|^{2}}{|x|^{2}} d x \\
& +C_{1} \chi \int_{\mathbb{R}^{3}} \frac{W(|x|)}{|x|}|u(x)|^{2} d x \leq C \int_{\mathbb{R}^{d}}|f(x)||\nabla u(x)| d x+C \int_{\mathbb{R}^{d}}|f(x)| \frac{|u(x)|}{|x|} d x \\
& +|\epsilon| \int_{\mathbb{R}^{d}}|u(x)||\nabla u(x)| d x+\left(\frac{1}{4} \chi(d)+(1-\chi(d))\right) \int_{\mathbb{R}^{d}} \frac{\nabla n(x) \cdot x}{|x|}|u(x)|^{2} d x .
\end{aligned}
$$

This inequality allows us to start proving: for any $\delta>0$ there exist two positive constants $A \equiv A(\delta)$ and $B \equiv B(\delta, \beta)$ such that

$$
\left\{\begin{array}{l}
A\|\nabla u\|_{X_{\rho}}^{2}+A \sup _{R \geq \rho} \frac{1}{R^{3}} \int_{B(0, R)}|u(x)|^{2} d x+A \chi(d) \int_{B(0, \rho)} \frac{|u(x)|^{2}}{|x|^{2}} d x \\
+A(d-3) \int_{\mathbb{R}^{d}} \frac{|u(x)|^{2}}{|x|^{3}} d x+(\alpha \chi(d)+(1-\chi(d))) \frac{\gamma}{2} \int_{\mathbb{R}^{d}} \frac{V(x)}{|x|}|u(x)|^{2} d x \\
+A \chi(d) \int_{\mathbb{R}^{3}} \frac{W(|x|)}{|x|}|u(x)|^{2} d x \leq B\left(\|f\|_{X_{\rho}^{*}}^{2}+\left\|\frac{f}{|n|^{\frac{1}{2}}}\right\|_{X_{\rho}^{*}}^{2}\right) \\
+\delta(|\epsilon|+\tau)\|u\|_{X_{\rho}}^{2}+\left(\frac{1}{4} \chi(d)+(1-\chi(d))\right)\left(\delta+\beta_{\rho}\right)\left\||n|^{\frac{1}{2}} u\right\|_{X_{\rho}}^{2},
\end{array}\right.
$$

that we will use in the proof of (75).

Proof of estimate (77)

We need to show that the right hand side of (76) is finite in order to take supremun in $R>\rho$. 
Take $\varphi=1$ in (106), from (8) and the fact that $V \geq 0$, we get

$$
\begin{gathered}
\int_{\mathbb{R}^{d}}|\nabla u(x)|^{2} d x \leq \int_{\mathbb{R}^{d}}\left|n ( x ) \left\|\left.u(x)\right|^{2} d x+\tau \int_{\mathbb{R}^{d}}|u(x)|^{2} d x+\int_{\mathbb{R}^{d}}|u \| f| d x\right.\right. \\
\leq\left(\left\|n_{1}\right\|_{\infty}+\tau+1\right)\|u\|_{L^{2}\left(\mathbb{R}^{d}\right)}^{2}+\|f\|_{L^{2}\left(\mathbb{R}^{d}\right)}^{2}+c_{1} \int_{\mathbb{R}^{d}}|\nabla u(x)|^{2} d x,
\end{gathered}
$$

with $0<c_{1}<1$, the constant in (8). From this, it follows that if $f \in L^{2}\left(\mathbb{R}^{d}\right)$, then $u$, the solution of (70), is in $W^{1,2}\left(\mathbb{R}^{d}\right)$.

From (78) and (8), it is easy to check that

$$
\left\||n|^{\frac{1}{2}} u\right\|_{X_{\rho}}^{2} \leq \frac{C\left(\left\|n_{1}\right\|_{\infty}, c_{1}\right)}{\rho}\left(\|u\|_{L^{2}\left(\mathbb{R}^{d}\right)}^{2}+\|\nabla u\|_{L^{2}\left(\mathbb{R}^{d}\right)}^{2}\right)<\infty .
$$

On the other hand, we have (37)-(39) and using (79) and (9), we have

$$
\begin{aligned}
& \int_{\mathbb{R}^{d}} \frac{\nabla n(x) \cdot x}{|x|}|u(x)|^{2} d x \leq \sum_{j \geq j_{0}} \int_{C_{j}} \frac{\nabla n(x) \cdot x}{|x||n(x)|}|n(x)||u(x)|^{2} d x \\
& \quad+\int_{B(0, \rho)} \frac{\nabla n(x) \cdot x}{|x||n(x)|}|n(x)||u(x)|^{2} d x \\
& \leq \sum_{j \geq j_{0}} \sup _{x \in C_{j}} \frac{\nabla n(x) \cdot x}{|x||n(x)|} \int_{C_{j}}|n(x)||u(x)|^{2} d x \\
& +\sup _{x \in B(0, \rho)} \frac{\nabla n(x) \cdot x}{|x||n(x)|} \int_{B(0, \rho)}|n(x)||u(x)|^{2} d x \leq \beta_{\rho}\left\||n|^{\frac{1}{2}} u\right\|_{X_{\rho}}^{2}<\infty .
\end{aligned}
$$

We can take the sup when $R \geq \rho$ in (76) to get

$$
\begin{gathered}
\|\nabla u\|_{X_{\rho}}^{2}+\sup _{R \geq \rho} \frac{1}{R^{3}} \int_{B(0, R)}|u(x)|^{2} d x+\int_{\mathbb{R}^{d}} \frac{V(x)}{|x|} d x \\
+(d-3) \int_{\mathbb{R}^{d}} \frac{|u(x)|^{2}}{|x|^{3}} d x+\chi(d) \int_{B(0, \rho)} \frac{|u(x)|^{2}}{|x|^{2}} d x+\chi \int_{\mathbb{R}^{3}} \frac{W(|x|)}{|x|}|u(x)|^{2} d x<\infty,
\end{gathered}
$$

and therefore the left hand side of (77) is finite.

Now let us see the a priori estimate (77). We study the terms on the right hand side of (76).

$$
C \int_{\mathbb{R}^{d}}\left|f(x)\|\nabla u(x) \mid d x \leq C\| f\left\|_{X_{\rho}^{*}}^{2}+\frac{C_{1}}{2}\right\| \nabla u \|_{X_{\rho}}^{2} .\right.
$$

Let $j_{0}$ be such that $2^{j_{0}}<\rho \leq 2^{j_{0}+1}$. Arguing as in (41), we have that

$$
\begin{gathered}
C \int_{\mathbb{R}^{d}}|f(x)| \frac{|u(x)|}{|x|} d x \leq C \int_{B(0, \rho)}|f(x)| \frac{|u(x)|}{|x|} d x+C \int_{|x|>\rho}|f(x)| \frac{|u(x)|}{|x|} d x \\
\leq C \int_{B(0, R)}|f(x)| \frac{|u(x)|}{|x|} d x+C\|f\|_{X_{\rho}^{*}}^{2}+\frac{C_{1}}{2} \sup _{R \geq \rho} \frac{1}{R^{3}} \int_{B(0, \rho)}|u(x)|^{2} d x,
\end{gathered}
$$

and

$$
\begin{array}{cl}
C \int_{B(0, \rho)}|f(x)| \frac{|u(x)|}{|x|} d x \leq C\|f\|_{X_{\rho}^{*}}^{2}+\frac{C_{1}(d-3)}{2} \int_{\mathbb{R}^{d}} \frac{|u(x)|^{2}}{|x|^{3}} d x & \text { for } d>3 \\
C \int_{B(0, \rho)}|f(x)| \frac{|u(x)|}{|x|} d x \leq C\|f\|_{X_{\rho}^{*}}^{2}+\frac{C_{1}}{2} \int_{B(0, \rho)} \frac{|u(x)|^{2}}{|x|^{2}} d x & \text { for } d=3 .
\end{array}
$$


Therefore, for $d \geq 3$ we have

$$
\begin{aligned}
& C \int_{\mathbb{R}^{d}}|f(x)| \frac{|u(x)|}{|x|} d x \leq C\|f\|_{X_{\rho}^{*}}^{2}+\frac{C_{1}}{2} \chi(d) \int_{B(0, \rho)} \frac{|u(x)|^{2}}{|x|^{2}} d x \\
& +\frac{C_{1}(d-3)}{2} \int_{\mathbb{R}^{d}} \frac{|u(x)|^{2}}{|x|^{3}} d x+\frac{C_{1}}{2} \sup _{R \geq \rho} \frac{1}{R^{3}} \int_{B(0, R)}|u(x)|^{2} d x .
\end{aligned}
$$

To control the third term on the right hand side of (76), use the first inequality of (78) to obtain

$$
\int_{\mathbb{R}^{d}}|\nabla u(x)|^{2} d x \leq\left(\tau+\left\|n_{1}\right\|_{L^{\infty}}\right) \int_{\mathbb{R}^{d}}|u(x)|^{2} d x+\int_{\mathbb{R}^{d}}|f(x) \| u(x)| d x+c_{1} \int_{\mathbb{R}^{d}}|\nabla u(x)|^{2} d x,
$$

and since $c_{1}<1$,

$$
|\epsilon| \int_{\mathbb{R}^{d}}|\nabla u(x)|^{2} d x \leq C\left(\tau+\left\|n_{1}\right\|_{L^{\infty}}\right)|\epsilon| \int_{\mathbb{R}^{d}}|u(x)|^{2} d x+C|\epsilon| \int_{\mathbb{R}^{d}}|f(x) \| u(x)| d x .
$$

Arguing as in the proof of (45) but replacing (44) by this last inequality, we obtain for $\delta>0$

$$
\begin{gathered}
|\epsilon| \int_{\mathbb{R}^{d}}\left|\nabla u(x)\left\|u(x)\left|d x \leq C\left(\tau+\left\|n_{1}\right\|_{L^{\infty}}+|\epsilon|\right)^{\frac{1}{2}} \int_{\mathbb{R}^{d}}\right| f(x)\right\| u(x)\right| d x \\
\quad \leq C\left(\left\|\frac{f}{|n|^{\frac{1}{2}}}\right\|_{X_{\rho}^{*}}^{2}+\|f\|_{X_{\rho}^{*}}^{2}\right)+\delta\left\|\left.|| n\right|^{\frac{1}{2}} u\right\|_{X_{\rho}}^{2}+\delta(\tau+|\epsilon|)\|u\|_{X_{\rho}}^{2} .
\end{gathered}
$$

From (76), using (82)- (84), and taking the sup in $R \geq \rho$, we get

$$
\begin{aligned}
& \sup _{R \geq \rho} \frac{C_{1}}{R^{3}} \int_{B(0, R)}|u(x)|^{2} d x+(\alpha \chi(d)+(1-\chi(d))) \frac{\gamma}{2} \int_{\mathbb{R}^{d}} \frac{V(x)}{|x|}|u(x)|^{2} d x \\
& +C_{1}(d-3) \int_{\mathbb{R}^{d}} \frac{|u(x)|^{2}}{|x|^{3}} d x+C_{1} \chi(d) \int_{B(0, \rho)} \frac{|u(x)|^{2}}{|x|^{2}} d x+C_{1} \chi(d) \int_{\mathbb{R}^{3}} \frac{W(|x|)}{|x|}|u(x)|^{2} d x \\
& +C_{1}\|\nabla u\|_{X_{\rho}}^{2} \leq C\left(\|f\|_{X_{\rho}^{*}}^{2}+\left\|\frac{f}{|n|^{\frac{1}{2}}}\right\|_{X_{\rho}^{*}}^{2}\right)+\frac{C_{1}(d-3)}{2} \int_{\mathbb{R}^{d}} \frac{|u(x)|^{2}}{|x|^{3}} d x \\
& +\frac{C_{1} \chi(d)}{2} \int_{B(0, \rho)} \frac{|u(x)|^{2}}{|x|^{2}} d x+\frac{C_{1}}{2} \sup _{R \geq \rho} \frac{1}{R^{3}} \int_{B(0, R)}|u(x)|^{2} d x+\frac{C_{1}}{2}\|\nabla u\|_{X_{\rho}}^{2} \\
& +\delta(|\epsilon|+\tau)\|u\|_{X_{\rho}}^{2}+\left(\frac{1}{4} \chi(d)+(1-\chi(d))\right)\left(\delta+\beta_{\rho}\right)\left\||n|^{\frac{1}{2}} u\right\|_{X_{\rho}}^{2},
\end{aligned}
$$

and (77) follows.

Proof of estimate (75). 
Fix $R \geq \rho$, if we argue as in the proof of (24), then we get an estimate which is similar to (54). More precisely, if $d \geq 3$ we obtain

$$
\begin{aligned}
& \frac{1}{2 R} \int_{B(0, R)}|\nabla u(x)|^{2} d x+\frac{(d-1)}{4 R^{2}} \int_{|x|=R}|u(x)|^{2} d \sigma_{R}(x) \\
& +\frac{\tau}{2 R} \int_{B(0, R)}|u(x)|^{2} d x+\frac{1}{2 R} \int_{B(0, R)}|n(x)||u(x)|^{2} d x+\frac{d-3}{4} \int_{|x|>R} \frac{|u(x)|^{2}}{|x|^{3}} d x \\
& +\frac{\gamma}{2 R} \int_{B(0, R)} V(x)|u(x)|^{2} d x+\frac{\gamma}{2} \int_{|x|>R} \frac{V(x)}{|x|}|u(x)|^{2} d x \\
& -(1-\eta) \frac{d-3}{4} \int_{\mathbb{R}^{d}} \frac{|u(x)|^{2}}{|x|^{3}} d x-\frac{\chi(d)}{2} \int_{\mathbb{R}^{3}} \frac{W(|x|)}{|x|}|u(x)|^{2} d x \\
& \leq \int_{\mathbb{R}^{d}}|f(x)||\nabla u(x)| d x+\frac{(d+1)}{2} \int_{\mathbb{R}^{d}}|f(x)| \frac{|u(x)|}{|x|} d x \\
& +|\epsilon| \int_{\mathbb{R}^{d}}|\nabla u(x)||u(x)| d x+\frac{1}{2} \int_{\mathbb{R}^{d}} \frac{V(x)}{|x|}|u(x)|^{2} d x+\frac{1}{2} \int_{\mathbb{R}^{d}} \frac{\nabla n(x) \cdot x}{|x|}|u(x)|^{2} d x .
\end{aligned}
$$

From (77)

$$
\int_{\mathbb{R}^{d}} \frac{V(x)}{|x|}|u(x)|^{2} d x+\chi(d) \int_{\mathbb{R}^{3}} \frac{W(|x|)}{|x|}|u(x)|^{2} d x<\infty .
$$

Then as in the proof of (777), we have

$$
\begin{gathered}
\|\nabla u\|_{X_{\rho}}^{2}+\sup _{R \geq \rho} \frac{1}{R^{2}} \int_{|x|=R}|u(x)|^{2} d \sigma_{R}(x)+\left\||n|^{\frac{1}{2}} u\right\|_{X_{\rho}}^{2}+\left\|V^{\frac{1}{2}} u\right\|_{X_{\rho}}^{2} \\
+\int_{\mathbb{R}^{d}} \frac{V(x)}{|x|}|u(x)|^{2} d x+\tau\|u\|_{X_{\rho}}^{2}+(d-3) \int_{\mathbb{R}^{d}} \frac{|u(x)|^{2}}{|x|^{3}} d x<\infty .
\end{gathered}
$$

In order to get the a priori estimate, we use (777), (80), (82), (83), (57), (58) and taking the sup in $R \geq \rho$ in (85), we have for an absolute constant $C_{2}$

$$
\begin{aligned}
& C_{2}\|\nabla u\|_{X_{\rho}}^{2}+\frac{C_{2}}{4} \sup _{R \geq \rho} \frac{1}{R^{2}} \int_{|x|=R}|u(x)|^{2} d \sigma_{R}(x)+\frac{1}{2}\left\||n|^{\frac{1}{2}} u\right\|_{X_{\rho}}^{2}+C_{2}\left\|V^{\frac{1}{2}} u\right\|_{X_{\rho}}^{2} \\
& +\frac{3 C_{2}}{4} \sup _{R \geq \rho} \frac{1}{R^{3}} \int_{B(0, R)}|u(x)|^{2} d x+\frac{\gamma}{2} \int_{\mathbb{R}^{d}} \frac{V(x)}{|x|}|u(x)|^{2} d x+\tau C_{2}\|u\|_{X_{\rho}}^{2} \\
& +(d-3) C_{2} \int_{\mathbb{R}^{d}} \frac{|u(x)|^{2}}{|x|^{3}} d x+C_{2} \chi(d) \int_{\mathbb{R}^{3}} \frac{W(|x|)}{|x|}|u(x)|^{2} d x \\
& \leq C\left(\|f\|_{X_{\rho}^{*}}^{2}+\left\|\frac{f}{|n|^{\frac{1}{2}}}\right\|_{X_{\rho}^{*}}^{2}\right)+\delta(|\epsilon|+\tau)\|u\|_{X_{\rho}}^{2}+\frac{C_{2}}{2} \sup _{R \geq \rho} \frac{1}{R^{3}} \int_{B(0, R)}|u(x)|^{2} d x \\
& +\frac{C_{2}}{2}\|\nabla u\|_{X_{\rho}}^{2}+\left(\frac{1}{4 \alpha} \chi(d)+(1-\chi(d))\left(\delta+2 \beta_{\rho}\right)\left\||n|^{\frac{1}{2}} u\right\|_{X_{\rho}}^{2} .\right.
\end{aligned}
$$

If $d>3$, we take $\delta$ small and since $\beta_{\rho}<1 / 4$, the terms

$$
\frac{C_{2}}{2}\|\nabla u\|_{X_{\rho}}^{2}, \quad \delta \tau\|u\|_{X_{\rho}}^{2}, \quad\left(\delta+2 \beta_{\rho}\right)\left\||n|^{\frac{1}{2}} u\right\|_{X_{\rho}}^{2} \quad \text { and } \quad \frac{C_{2}}{2} \sup _{R \geq \rho} \frac{1}{R^{3}} \int_{B(0, R)}|u(x)|^{2} d x
$$

can be absorbed by their analogous on the left hand side of the above inequality in order to obtain (75). 
If $d=3$, we will need that

$$
\beta_{\rho}<\alpha<\frac{1}{2}-\int_{0}^{\infty} t W(t) d t
$$

The proof of (72) is similar to the previous one, but in this case, we have to replace $C\left(\|f\|_{X_{\rho}^{*}}^{2}+\left\||n|^{-1 / 2}|f|\right\|_{X_{\rho}^{*}}^{2}\right)$ by $C\left(\tau_{0}\right)\|f\|_{X_{\rho}^{*}}^{2}$ for all $\tau \geq \tau_{0}$. In order to do this, we have to modify the estimate (84) as follows:

$$
\begin{aligned}
& |\epsilon| \int_{\mathbb{R}^{d}}\left|\nabla u(x)\left\|u(x)\left|d x \leq C\left(\tau+\left\|n_{1}\right\|_{L^{\infty}}+|\epsilon|\right)^{\frac{1}{2}} \int_{\mathbb{R}^{d}}\right| f(x)\right\| u(x)\right| d x \\
& \leq C\left(\frac{\left\|n_{1}\right\|_{L^{\infty}}}{\tau_{0}}\right)^{\frac{1}{2}}\|f\|_{X_{\rho}^{*}}\left\||n|^{\frac{1}{2}} u\right\|_{X_{\rho}}+C\left(\tau_{0}\right)(\tau+|\epsilon|)^{\frac{1}{2}}\|f\|_{X_{\rho}^{*}}\|u\|_{X_{\rho}} \quad \tau \geq \tau_{0} .
\end{aligned}
$$

3.2. Proof of Theorem 1.2. We start with the proof of estimate (10). Estimate (11) is treated in a similar way.

Following the proof of the Theorem [1.1, for $R \geq \rho$ fixed, we consider the function $\psi_{R}$ defined by (59).

As in the proof of (5), it suffices to prove that the operator $\psi_{R}^{\frac{1}{2}} D^{\frac{1}{2}}$ is $H$-supersmooth on $\left[\tau_{0}, \infty\right)\left(\psi_{R}^{\frac{1}{2}} D^{\frac{1}{2}} P_{\left[\tau_{0}, \infty\right)}\right.$ is $H$-supersmooth, see [15], v IV pag.163), which means that for all $f \in D\left(D^{\frac{1}{2}} \psi_{R}^{\frac{1}{2}}\right) \subset L^{2}\left(\mathbb{R}^{d}\right), \tau \geq \tau_{0}$ and $\epsilon>0$, there exists a positive constant $C\left(\beta_{\rho}, \tau_{0}\right)$ independent of $\tau, \epsilon$ and $R$ such that

$$
\left\|\psi_{R}^{\frac{1}{2}} D^{\frac{1}{2}} R_{H}(\tau \pm i \epsilon) D^{\frac{1}{2}} \psi_{R}^{\frac{1}{2}} f\right\|_{L^{2}} \leq C\left(\beta_{\rho}, \tau_{0}\right)\|f\|_{L^{2}} .
$$

(Here, we use that the operators $e^{i t H}$ and $P_{\tau_{0}}$ commute).

Arguing as in the proof of Theorem 1.1, but using (73) instead (20), we obtain (10).

(11) follows in a similar way.

\section{Potentials Without smallness assumptions}

4.1. Estimates for the resolvent. As in the previous sections we start with the Helmholtz equation.

Proposition 4.1. Let $V_{1}$ and $V_{2}$ be two real valued potentials as in Theorem 1.3, and let $u$ be a solution of the equation

$$
-\Delta u+\left(V_{1}+V_{2}\right) u \pm i \epsilon u+\tau u=f, \quad \epsilon \neq 0, x \in \mathbb{R}^{d} \quad d \geq 2 .
$$

- If $d \geq 3$, given $\tau_{0}>0$ there exits $B\left(\tau_{0}\right)$ such that

$$
\tau_{0} B\left(\tau_{0}\right)=o(1) \quad \tau_{0} \rightarrow 0
$$


and the following a priori estimate holds:

$$
\begin{array}{cc}
\|\nabla u\|_{X_{\tau_{0}}}^{2}+\tau\|u\|_{X_{\tau_{0}}}^{2}+(d-3) \int_{|x|>\tau_{0}} \frac{|u(x)|^{2}}{|x|^{3}} d x & \\
+\sup _{R \geq \tau_{0}} \frac{1}{R^{2}} \int_{|x|=R}|u(x)|^{2} d \sigma_{R}(x) \leq C\|f\|_{X_{\tau_{0}}^{*}}^{2} \quad \text { for } \tau \geq B\left(\tau_{0}\right) .
\end{array}
$$

- If $d=2$, there exists $\tau_{0}>0$ and $B\left(\tau_{0}\right)$ satisfying 87 and $u$ verifies the following a priori estimate:

$$
\begin{array}{rr}
\|\nabla u\|_{X_{\tau_{0}}}^{2}+\tau\|u\|_{X_{\tau_{0}}}^{2}+\sup _{R \geq \tau_{0}} \frac{1}{R^{2}} \int_{|x|=R}|u(x)|^{2} d \sigma_{R}(x) \\
\leq C\|f\|_{X_{\tau_{0}}^{*}}^{2} \quad \text { for } \tau \geq B\left(\tau_{0}\right) .
\end{array}
$$

Here, $C$ is an absolute positive constant independent of $\epsilon, \tau$ and $\tau_{0}$.

Proof. By a density argument we can assume without loss of generality that $f \in$ $L^{2}\left(\mathbb{R}^{d}\right)$.

We begin with the case $d \geq 3$. For $R>\tau_{0}$ fixed, we consider the functions $\Phi_{R}$ and $\varphi_{R}$ defined by (50) and (51). If we put $\Phi=\Phi_{R}$ and $\varphi=\varphi_{R}$ in (111), arguing as we did to get (54), we obtain

$$
\begin{gathered}
\frac{1}{2 R} \int_{B(0, R)}|\nabla u(x)|^{2} d x+\frac{\tau}{2 R} \int_{B(0, R)}|u(x)|^{2} d x \\
+\frac{(d-1)(d-3)}{4} \int_{|x|>R} \frac{|u(x)|^{2}}{|x|^{3}} d x+\frac{(d-1)}{8 R^{2}} \int_{|x|=R}|u(x)|^{2} d \sigma_{R}(x) \\
+\leq \frac{1}{2} \int_{\mathbb{R}^{d}}\left|\nabla V_{1}(x) \cdot \nabla \Phi_{R}(x)\right||u(x)|^{2} d x+\frac{1}{2} \int_{\mathbb{R}^{d}}\left|V_{2}(x)\right|\left|\nabla\left(|u|^{2}\right)(x)\right| d x \\
+\frac{1}{2} \int_{\mathbb{R}^{d}} \varphi_{R}(x)\left|V_{1}(x)+V_{2}(x)\right||u(x)|^{2} d x+\frac{1}{2} \int_{\mathbb{R}^{d}}\left|V_{2}(x)\right|\left|\Delta \Phi_{R}(x)\right||u(x)|^{2} d x \\
+\int_{\mathbb{R}^{d}}|f(x)||\nabla u(x)| d x+\int_{\mathbb{R}^{d}} \varphi_{R}(x)|f(x)||u(x)| d x \\
+\frac{1}{2} \int_{\mathbb{R}^{d}}|f(x)|\left|\Delta \Phi_{R}(x)\right||u(x)| d x+|\epsilon| \int_{\mathbb{R}^{d}}|u(x)||\nabla u(x)| d x .
\end{gathered}
$$

As in the proof of theorem 2.1 and theorem 3.1 and using (12)-(14), we can see that the left hand side of (90) is independent of $R \geq \tau_{0}$ and it is finite. Then, taking the sup in $R \geq \tau_{0}$ on the right hand side of (90), we have

$$
\begin{gathered}
\|\nabla u\|_{X_{\tau_{0}}}^{2}+\tau\|u\|_{X_{\tau_{0}}}^{2} \\
+(d-3) \int_{|x|>\tau_{0}} \frac{|u(x)|^{2}}{|x|^{3}} d x+\sup _{R \geq \tau_{0}} \frac{1}{R^{2}} \int_{|x|=R}|u(x)|^{2} d \sigma_{R}(x)<\infty .
\end{gathered}
$$

To prove the a priori estimate, we study the terms on the left hand side of (90). $C$ will denote an absolute constant independent of $\tau, \tau_{0}, \epsilon$ and $a$ (the constant in (12)-(14) ). 
It is easy to check

$$
\begin{gathered}
\int_{\mathbb{R}^{d}}\left|\nabla V_{1}(x) \cdot \nabla \Phi_{R}(x)\right||u(x)|^{2} d x \leq a\left(1+\frac{2 \gamma}{1-2^{\gamma}} \max \left\{1, \frac{1}{\tau_{0}}-1\right\}\right)\|u\|_{X_{\tau_{0}}}^{2}, \\
\int_{\mathbb{R}^{d}}\left|V_{2}(x)\right|\left|\nabla\left(|u|^{2}\right)(x)\right| d x \leq 2 a^{2}\|u\|_{X_{\tau_{0}}}^{2}+\frac{1}{8}\|\nabla u\|_{X_{\tau_{0}}}^{2}, \\
\frac{1}{2} \int_{\mathbb{R}^{d}} \varphi_{R}(x)\left|V_{1}(x)+V_{2}(x)\right||u(x)|^{2} d x \leq a\|u\|_{X_{\tau_{0}}}^{2}
\end{gathered}
$$

(94) $\frac{1}{2} \int_{\mathbb{R}^{d}}\left|V_{2}(x)\right|\left|\Delta \Phi_{R}(x)\right||u(x)|^{2} \leq \frac{a d}{2}\left(1+\frac{2 \gamma}{1-2^{\gamma}} \max \left\{1, \frac{1}{\tau_{0}}-1\right\}\right)\|u\|_{X_{\tau_{0}}}^{2}$,

$$
\begin{aligned}
& \int_{\mathbb{R}^{d}} \varphi_{R}(x)|f(x)||u(x)| d x+\int_{\mathbb{R}^{d}}|f(x)|\left|\Delta \Phi_{R}(x)\right||u(x)| d x \\
& \leq \frac{d}{R} \int_{B(0, R)}|f(x)||u(x)| d x+(d-1) \int_{|x|>R} \frac{1}{|x|}|f(x)||u(x)| d x \\
& \leq d^{2} C(\delta)\|f\|_{X_{\tau_{0}}^{*}}^{2}+\|u\|_{X_{\tau_{0}}}^{2},
\end{aligned}
$$

and as in the proof of estimate (77)

$$
\begin{gathered}
|\epsilon| \int_{\mathbb{R}^{d}}|\nabla u(x)||u(x)| d x \leq C\|f\|_{X_{\tau_{0}}^{*}}^{2}+\delta(\tau+1)\|u\|_{X_{\tau_{0}}}^{2} \\
\quad+\frac{1}{8}\|\nabla u\|_{X_{\tau_{0}}}+\frac{d-1}{16} \sup _{R \geq \tau_{0}} \frac{1}{R^{3}} \int_{B(0, R)}|u(x)|^{2} d x .
\end{gathered}
$$

Let us define

$$
B\left(\tau_{0}\right)=16 a\left(d^{2}-1\right)\left(1+\frac{2^{\gamma}}{1-2^{\gamma}} \max \left\{1, \frac{1}{\tau_{0}}-1\right\}\right)+a(2 a+1) .
$$

From (90), using (91)-(96) and taking

$$
\delta<\min \left\{\frac{B\left(\tau_{0}\right)}{4}, \frac{1}{4}\right\}
$$

we get

$$
\begin{gathered}
\frac{1}{R} \int_{B(0, R)}|\nabla u(x)|^{2} d x+\frac{\tau}{R} \int_{B(0, R)}|u(x)|^{2} d x \\
+(d-3) \int_{|x|>R} \frac{|u(x)|^{2}}{|x|^{3}} d x+\frac{1}{R^{2}} \int_{|x|=R}|u(x)|^{2} d \sigma_{R}(x) \\
\leq C\|f\|_{X_{\tau_{0}}^{*}}^{2}+\left(\frac{\tau}{2}+\frac{B\left(\tau_{0}\right)}{4}\right)\|u\|_{X_{\tau_{0}}}^{2}+\frac{1}{2}\|\nabla u\|_{X_{\tau_{0}}}^{2}+\frac{d-1}{16} \sup _{R \geq \tau_{0}} \frac{1}{R^{3}} \int_{B(0, R)}|u(x)|^{2} d x .
\end{gathered}
$$

If $\tau>B\left(\tau_{0}\right)$, by taking the sup in the above inequality and using (57), the terms

$$
\left(\frac{\tau}{2}+\frac{B\left(\tau_{0}\right)}{4}\right)\|u\|_{X_{\tau_{0}}}^{2}, \quad \frac{1}{2}\|\nabla u\|_{X_{\tau_{0}}}^{2} \quad \text { and } \quad \frac{d-1}{16} \sup _{R \geq \tau_{0}} \frac{1}{R^{3}} \int_{B(0, R)}|u(x)|^{2} d x
$$

on the right hand side can be passed to the l.h.s. and we get (88). 
Id $d=2$, we write (90) as

$$
\begin{gathered}
\frac{1}{2 R} \int_{B(0, R)}|\nabla u(x)|^{2} d x+\frac{\tau}{2 R} \int_{B(0, R)}|u(x)|^{2} d x+\frac{1}{8 R^{2}} \int_{|x|=R}|u(x)|^{2} d \sigma_{R}(x) \\
\leq \frac{1}{2} \int_{\mathbb{R}^{2}} \nabla V_{1}(x) \cdot \nabla \Phi_{R}(x)|u(x)|^{2} d x+\frac{1}{2} \int_{\mathbb{R}^{2}}\left|V_{2}(x)\right|\left|\nabla\left(|u|^{2}\right)(x)\right| d x \\
(98)+\left.\frac{1}{2}\left|\int_{\mathbb{R}^{2}} \varphi_{R}(x)\left(V_{1}(x)+V_{2}(x)\right)\right| u(x)\right|^{2} d x\left|+\frac{1}{2}\right| \int_{\mathbb{R}^{2}} V_{2}(x) \Delta \Phi_{R}(x)|u(x)|^{2} d x \mid \\
\quad+\int_{\mathbb{R}^{2}}|f(x)||\nabla u(x)| d x+\left|\int_{\mathbb{R}^{2}} \varphi_{R}(x) f(x) \bar{u}(x) d x\right| \\
+\frac{1}{2}\left|\int_{\mathbb{R}^{2}} f(x) \Delta \Phi_{R}(x) \bar{u}(x) d x\right|+|\epsilon| \int_{\mathbb{R}^{2}}|u(x)||\nabla u(x)| d x+\frac{1}{4} \int_{|x|>R} \frac{|u(x)|^{2}}{|x|^{3}} d x .
\end{gathered}
$$

Let us study the last term in the above inequality.

Using (97), we define $\tau_{0}$, and therefore $B\left(\tau_{0}\right)$, such that

$$
\frac{1}{2 B\left(\tau_{0}\right) \tau_{0}^{2}}<1
$$

and let us take $j_{0}$ satisfying $2^{j_{0}}<\tau_{0} \leq 2^{j_{0}+1}$.

We know, for $R>\tau_{0}$, that

$$
\frac{1}{4} \int_{|x|>R} \frac{|u(x)|^{2}}{|x|^{3}} d x \leq \frac{1}{4 \tau_{0}^{3}} \int_{|x|>\tau_{0}}|u(x)|^{2} d x<\infty
$$

and

$$
\frac{1}{4} \int_{|x|>R} \frac{|u(x)|^{2}}{|x|^{3}} d x \leq \frac{1}{4} \sum_{j \geq j_{0}} \int_{|x| \sim 2^{j}} \frac{|u(x)|^{2}}{|x|^{3}} d x \leq \frac{1}{4 \tau_{0}^{2}}\|u\|_{X_{\tau_{0}}}^{2} \leq \frac{B\left(\tau_{0}\right)}{2}\|u\|_{X_{\tau_{0}}}^{2} .
$$

Then (89) follows as in the case $d \geq 3$ since the term $\frac{1}{4} \int_{|x|>R} \frac{|u(x)|^{2}}{|x|^{3}} d x$ can be taken to the l.h.s. and can be absorbed by $\tau\|u\|_{X_{\tau_{0}}}^{2}$ if $\tau \geq B\left(\tau_{0}\right)$.

Remark 4.2. Suppose that $\tau_{0}=1$ in Proposition 4.1 and $d \geq 3$, then

$$
B(a) \equiv B(1)=16 a\left(d^{2}-1\right)\left(1+\frac{2^{\gamma}}{1-2^{\gamma}}\right)+a(2 a+1) .
$$

Let be $\eta>0$ and assume that $a$, the constant in (12)-(14), satisfies that $B(a) \leq \eta$. Then, by Proposition 4.1, we have

$$
\|\nabla u\|_{X_{1}}^{2}+\tau\|u\|_{X_{1}}^{2} \leq C\|f\|_{X_{1}^{*}}^{2}, \quad \tau \geq \eta .
$$

and if we follow the lines of the proof of Theorem 1.2 we can deduce

$$
\sup _{R \geq 1} \frac{1}{R} \int_{B(0, R)} \int_{-\infty}^{\infty}\left|D^{\frac{1}{2}} e^{i t H} \mathcal{P}_{\eta} u_{0}(x)\right|^{2} d t d x \leq C\left\|u_{0}\right\|_{2}^{2}
$$

and

$$
\tau \sup _{R \geq 1} \frac{1}{R} \int_{B(0, R)} \int_{-\infty}^{\infty}\left|e^{i t H} \mathcal{P}_{\eta} u_{0}(x)\right|^{2} d t d x \leq C\left\|u_{0}\right\|_{2}^{2} \quad \tau \geq \eta
$$


Lemma 4.3. (Ikebe-Saito) Let $\tau_{0}$ and $\tau_{1}$ two positive real numbers such that $\tau_{0}<\tau_{1}$ and $\alpha>0$. Let us consider the sequences

$$
\left\{\tau_{n}+i \epsilon_{n}\right\}_{n \in \mathbb{N}} \quad \tau_{0}<\tau_{n}<\tau_{1} \quad \text { and } \quad 0<\epsilon_{n}<1,
$$

$\left\{f_{n}\right\}_{n \in \mathbb{N}} \in L^{2}\left((1+|x|)^{1+\alpha}\right.$ and $u_{n} \in L^{2}\left((1+|x|)^{-1-\alpha}\right)$ the solution of

$$
-\Delta u+\left(V_{1}+V_{2}\right) u \pm i \epsilon_{n} u+\tau_{n} u=f_{n}, \quad x \in \mathbb{R}^{d} \quad d \geq 2 .
$$

with $V_{1}$ and $V_{2}$ satisfying the conditions of Theorem 1.3 .

If $f_{n} \rightarrow f$ in $L^{2}\left((1+|x|)^{1+\alpha}\right.$ and $\tau_{n}+i \epsilon_{n} \rightarrow \tau+i \epsilon$, then there exists $\lim _{n \rightarrow \infty} u_{n}=$ $u$ in $L^{2}\left((1+|x|)^{-1-\alpha}\right.$ and such that $u$ is the unique solution of

$$
-\Delta u+\left(V_{1}+V_{2}\right) u \pm i \epsilon u+\tau u=f, \quad x \in \mathbb{R}^{d} \quad d \geq 2 .
$$

Proof. This lemma is a consequence of Theorem 1.3, Lemma 1.11 and Theorem 1.4 of 8 .

Proposition 4.4. Let $\tau_{0}, \tau_{1}, \alpha, V_{1}$ and $V_{2}$ as in lemma 4.3 and $f \in L^{2}((1+$ $\left.|x|)^{1+\alpha}\right)$. Then, the solution $u \in L^{2}\left((1+|x|)^{-1-\alpha}\right)$ of

$$
-\Delta u+\left(V_{1}+V_{2}\right) u \pm i \epsilon+\tau u=f, \quad x \in \mathbb{R}^{d} \quad 0<\epsilon<1 \quad d \geq 2,
$$

satisfies, for $\tau_{0}<\tau<\tau_{1}$, the a priori estimate

$$
\|u\|_{L^{2}\left((1+|x|)^{-1-\alpha}\right)}+\|\nabla u\|_{L^{2}\left((1+|x|)^{-1-\alpha}\right)} \leq C\|f\|_{L^{2}\left((1+|x|)^{1+\alpha}\right)}^{2},
$$

where $C$ is a constant that only depends on $\tau_{0}$, and $\tau_{1}$.

Proof. Suppose that (99) is false. Then, on one hand, there exist sequences $\left\{\epsilon_{n}\right\} \in$ $(0,1),\left\{\tau_{n}\right\} \in\left(\tau_{0}, \tau_{1}\right),\left\{f_{n}\right\} \in L^{2}\left((1+|x|)^{1+\alpha}\right)$ and $\left\{u_{n}\right\} \in L^{2}\left((1+|x|)^{-1-\alpha}\right)$ solution of

$$
-\Delta u+\left(V_{1}+V_{2}\right) u \pm i \epsilon_{n}+\tau_{n} u=f_{n}, \quad x \in \mathbb{R}^{d} \quad d \geq 2,
$$

such that

$$
\lim _{n \rightarrow 0}\left\|f_{n}\right\|_{L^{2}\left((1+|x|)^{1+\alpha}\right)}=0
$$

and for all $n \in \mathbb{N}$,

$$
\left\|u_{n}\right\|_{L^{2}\left((1+|x|)^{-1-\alpha}\right)}+\left\|\nabla u_{n}\right\|_{L^{2}\left((1+|x|)^{-1-\alpha}\right)}=1 .
$$

We can take subsequence $\tau_{0}<\tau_{m}<\tau_{1}$ and $0<\epsilon_{m}<1$ such that

$$
\tau_{m}+i \epsilon_{m} \rightarrow \tau+i \epsilon
$$

with $\tau_{0} \leq \tau \leq \tau_{1}$ and $0 \leq \epsilon \leq 1$. Since $f_{m} \rightarrow 0$ in $L^{2}\left((1+|x|)^{1+\alpha}\right)$, from Ikebe-Saito's lemma $u_{m} \rightarrow 0$ in $L^{2}\left((1+|x|)^{-1-\alpha}\right)$.

On the other hand, if we take $\varphi=\frac{1}{(1+|x|)^{1+\alpha}}$ in (106) and we use (12) and (13), we obtain

$$
\begin{gathered}
\int_{\mathbb{R}^{d}} \frac{\left|\nabla u_{m}(x)\right|^{2}}{(1+|x|)^{1+\alpha}} d x \leq \\
C\left(1+\tau_{m}\right) \int_{\mathbb{R}^{d}} \frac{\left|u_{m}(x)\right|^{2}}{(1+|x|)^{1+\alpha}} d x+C\left\|f_{m}\right\|_{L^{2}\left((1+|x|)^{1+\alpha}\right)}\left\|u_{m}\right\|_{L^{2}\left((1+|x|)^{-1-\alpha}\right)},
\end{gathered}
$$

then

$$
\int_{\mathbb{R}^{d}} \frac{\left|\nabla u_{m}(x)\right|^{2}}{(1+|x|)^{1+\alpha}} d x \longrightarrow 0
$$


but this is a contradiction with the fact that $u_{m} \rightarrow 0$ in $L^{2}\left((1+|x|)^{-1-\alpha}\right)$ and (101).

Theorem 4.5. Let $V_{1}$ and $V_{2}$ as in proposition 4.1 and $u$ the solution of (86). Given $\tau_{0}>0$ and $\alpha>0$, the following a priori estimate holds:

$$
\|\nabla u\|_{L^{2}\left((1+|x|)^{-1-\alpha}\right)}^{2}+\tau\|u\|_{L^{2}\left((1+|x|)^{-1-\alpha}\right)}^{2} \leq C\left(\tau_{0}\right)\|f\|_{L^{2}\left((1+|x|)^{1+\alpha}\right)}^{2},
$$

where $C\left(\tau_{0}\right)$ is a positive constant independent of $\epsilon$ and $\tau$.

Proof. Again, by a density argument we can assume without loss of generality that $f \in L^{2}\left(\mathbb{R}^{d}\right)$.

For $\tau_{0}>0$ we have:

$$
L^{2}\left((1+|x|)^{1+\alpha}\right) \subset X_{\tau_{0}}^{*} \quad \text { and } \quad\|f\|_{X_{\tau_{0}}^{*}} \leq C \tau_{0}^{\frac{1}{2}}\|f\|_{L^{2}\left((1+|x|)^{1+\alpha}\right)}
$$

and

$$
X_{\tau_{0}} \subset L^{2}\left((1+|x|)^{-1-\alpha}\right) \quad \text { and } \quad\|u\|_{L^{2}\left((-1-|x|)^{1+\alpha}\right)} \leq C \tau_{0}^{\frac{1}{2}}\|u\|_{X_{\tau_{0}}} .
$$

Let $B\left(\tau_{0}\right)$ be the constant defined by (97). If $B\left(\tau_{0}\right) \leq \tau_{0}$, (102) is a consequence of (103), (104) and (88) ( or (89)) .

Suppose that $B\left(\tau_{0}\right)>\tau_{0}$. If $\tau \in\left[\tau_{0}, B\left(\tau_{0}\right)\right]$, (102) is a consequence of proposition 4.4. If $\tau \geq B\left(\tau_{0}\right)$, (102) is a consequence of proposition 4.1.

4.2. Proof of the theorem 1.3. We prove estimate (15) and (16) can be obtained in a similar way.

We write $\psi(x)=(1+|x|)^{-1-\alpha}$. To show that (15) holds it is enough to prove that the operator $\psi^{\frac{1}{2}} D^{\frac{1}{2}}$ is $H$-supersmooth in $\left[\tau_{0}, \infty\right)$, which means by definition that for all $f \in \mathcal{D}\left(\psi^{\frac{1}{2}} D^{\frac{1}{2}}\right) \subset L^{2}\left(\mathbb{R}^{d}\right), \tau \geq \tau_{0}$ and $\epsilon>0$, there exists a positive constant $C$ independent of $\tau$ and $\epsilon$ such that

$$
\left\|\psi^{\frac{1}{2}} D^{\frac{1}{2}} R_{H}(\tau \pm i \epsilon) D^{\frac{1}{2}} \psi^{\frac{1}{2}} f\right\|_{L^{2}} \leq C\left(\tau_{0}\right)\|f\|_{L^{2}} .
$$

Following the proof of theorem 1.1, but using theorem 4.5] instead of (20), we get (15).

\section{Appendix 1}

Here we state some identities that have been used throughout the paper. They follow by using integration by parts.

Let $u$ be a solution of the equation

$$
-\Delta u+V(x) u \pm i \epsilon u-\tau u=f, \quad \epsilon \neq 0, \quad x \in \mathbb{R}^{d} \quad d \geq 2 .
$$


If $\varphi$ and $\Phi$ are two real valued functions in $\mathcal{S}\left(\mathbb{R}^{d}\right)$, then the following identities hold:

$$
\mid \begin{gathered}
\int_{\mathbb{R}^{d}} \varphi(x)|\nabla u(x)|^{2} d x-\frac{1}{2} \int_{\mathbb{R}^{d}} \Delta \varphi(x)|u(x)|^{2} d x+\int_{\mathbb{R}^{d}} \varphi(x) V(x)|u(x)|^{2} d x \\
-\tau \int_{\mathbb{R}^{d}} \varphi(x)|u(x)|^{2} d x=\Re \int_{\mathbb{R}^{d}} \varphi(x) f(x) \bar{u}(x) d x
\end{gathered}
$$

$$
\pm \epsilon \int_{\mathbb{R}^{d}} \varphi(x)|u(x)|^{2} d x+\Im \int_{\mathbb{R}^{d}} \nabla \varphi(x) \cdot \nabla u(x) \bar{u}(x) d x=\Im \int_{\mathbb{R}^{d}} \varphi(x) f(x) \bar{u}(x) d x
$$

$$
\begin{aligned}
& \int_{\mathbb{R}^{d}} \nabla \bar{u}(x) \cdot D^{2} \Phi(x) \cdot \nabla u(x) d x-\frac{1}{4} \int_{\mathbb{R}^{d}} \Delta^{2} \Phi(x)|u(x)|^{2} d x \\
& \quad-\frac{1}{2} \int_{\mathbb{R}^{d}} \nabla V(x) \cdot \nabla \Phi(x)|u(x)|^{2} d x= \pm \epsilon \Im \int_{\mathbb{R}^{d}} \nabla \Phi(x) \cdot \nabla \bar{u}(x) u(x) d x \\
& \quad-\Re \int_{\mathbb{R}^{d}} f(x)\left(\nabla \Phi(x) \cdot \nabla \bar{u}(x)+\frac{1}{2} \Delta \Phi(x) \bar{u}(x)\right) d x .
\end{aligned}
$$

$$
\mid \begin{aligned}
& \int_{\mathbb{R}^{d}} \nabla \bar{u}(x) \cdot D^{2} \Phi(x) \cdot \nabla u(x) d x-\int_{\mathbb{R}^{d}} \varphi(x)|\nabla u(x)|^{2} d x \\
& +\frac{1}{4} \int_{\mathbb{R}^{d}} \Delta(2 \varphi-\Delta \Phi(x))|u(x)|^{2} d x-\frac{1}{2} \int_{\mathbb{R}^{d}} \nabla V(x) \cdot \nabla \Phi(x)|u(x)|^{2} d x \\
& -\int_{\mathbb{R}^{d}} \varphi(x) V(x)|u(x)|^{2} d x+\tau \int_{\mathbb{R}^{d}} \varphi(x)|u(x)|^{2} d x \\
& =-\Re \int_{\mathbb{R}^{d}} \varphi(x) f(x) \bar{u}(x) d x \pm \epsilon \Im \int_{\mathbb{R}^{d}} \nabla \Phi(x) \cdot \nabla \bar{u}(x) u(x) d x \\
& -\Re \int_{\mathbb{R}^{d}} f(x)\left(\nabla \Phi(x) \cdot \nabla \bar{u}(x)+\frac{1}{2} \Delta \Phi(x) \bar{u}(x)\right) d x .
\end{aligned}
$$

Furthermore, if we write $V(x)=V_{1}(x)+V_{2}(x)$, then

$$
\mid \begin{aligned}
& \int_{\mathbb{R}^{d}} \nabla \bar{u}(x) \cdot D^{2} \Phi(x) \cdot \nabla u(x) d x \\
& -\frac{1}{4} \int_{\mathbb{R}^{d}} \Delta^{2} \Phi(x)|u(x)|^{2} d x-\frac{1}{2} \int_{\mathbb{R}^{d}} \nabla V_{1}(x) \cdot \nabla \Phi(x)|u(x)|^{2} d x \\
& +\frac{1}{2} \int_{\mathbb{R}^{d}} V_{2}(x) \nabla \Phi(x) \cdot \nabla\left(|u(x)|^{2}\right) d x+\frac{1}{2} \int_{\mathbb{R}^{d}} V_{2}(x) \Delta \Phi(x)|u(x)|^{2} d x \\
& =-\Re \int_{\mathbb{R}^{d}} f(x)\left(\nabla \Phi(x) \cdot \nabla \bar{u}(x)+\frac{1}{2} \Delta \Phi(x) \bar{u}(x)\right) d x \\
& \pm \epsilon \Im \int_{\mathbb{R}^{d}} \nabla \Phi(x) \cdot \nabla \bar{u}(x) u(x) d x .
\end{aligned}
$$

and

$$
\mid \begin{aligned}
& \int_{\mathbb{R}^{d}} \nabla \bar{u}(x) \cdot D^{2} \Phi(x) \cdot \nabla u(x) d x-\int_{\mathbb{R}^{d}} \varphi(x)|\nabla u(x)|^{2} d x \\
& +\frac{1}{4} \int_{\mathbb{R}^{d}} \Delta(2 \varphi-\Delta \Phi(x))|u(x)|^{2} d x-\frac{1}{2} \int_{\mathbb{R}^{d}} \nabla V_{1}(x) \cdot \nabla \Phi(x)|u(x)|^{2} d x \\
& +\frac{1}{2} \int_{\mathbb{R}^{d}} V_{2}(x) \Delta \Phi(x)|u(x)|^{2} d x+\frac{1}{2} \int_{\mathbb{R}^{d}} V_{2}(x) \nabla \Phi(x) \cdot \nabla\left(|u(x)|^{2}\right) d x \\
& -\int_{\mathbb{R}^{d}} \varphi(x) V(x)|u(x)|^{2} d x+\tau \int_{\mathbb{R}^{d}} \varphi(x)|u(x)|^{2} d x \\
& =-\Re \int_{\mathbb{R}^{d}} \varphi(x) f(x) \bar{u}(x) d x \pm \epsilon \Im \int_{\mathbb{R}^{d}} \nabla \Phi(x) \cdot \nabla \bar{u}(x) u(x) d x \\
& -\Re \int_{\mathbb{R}^{d}} f(x)\left(\nabla \Phi(x) \cdot \nabla \bar{u}(x)+\frac{1}{2} \Delta \Phi(x) \bar{u}(x)\right) d x,
\end{aligned}
$$

where $\Delta^{2}$ denotes the bilaplacian and $D^{2} \Phi$ denotes the Hessian matrix of $\Phi$ with respect to $x_{1}, \ldots, x_{d}$. 


\section{Appendix 2}

The following lemma is implicit in [3].

Lemma 6.1. Let $\alpha, \epsilon, \kappa$ and $R$ four positive constants and $h(t)$ a no negative function in $(0, \infty)$ such that

$$
\alpha+\frac{\epsilon}{6}+\int_{0}^{\infty} t h(t) d t<\kappa<\frac{1}{2} .
$$

Then, we can find a radial function $\Phi(x) \equiv \Phi(r),|x|=r$, solution of

$$
\Delta^{2} \Phi(x)=-\frac{\epsilon}{R^{3}} \chi_{(0, R)}(x)-\frac{h(|x|)}{|x|} \quad x \in \mathbb{R}^{3}
$$

such that

$$
\begin{gathered}
\inf _{r>0}\left\{\Phi^{\prime}(r), \Phi^{\prime \prime}(r)\right\} \geq 0, \\
\inf _{r \in(0, R)}\left\{\frac{\Phi^{\prime}(r)}{r}, \Phi^{\prime \prime}(r)\right\} \geq \frac{C \epsilon}{R}, \\
\alpha<\Phi^{\prime}(r)<\kappa<\frac{1}{2}, \quad r>0,
\end{gathered}
$$

where $C$ is an absolute constant.

Proof. In dimension three the bilaplacian of a radial function $\Phi$ has the simple expression $\Phi^{i v}+\frac{4}{r} \Phi^{i i i}$.

If we integrate (113) we have

$$
\Phi^{\prime}(r)=\psi^{\prime}(r)+\varphi^{\prime}(r)
$$

where

$$
\begin{gathered}
\psi^{\prime}(r)=\frac{1}{r^{2}} \int_{0}^{r} u^{2} \int_{u}^{\infty} \frac{1}{s^{2}} \int_{0}^{s} t h(t) d t d s d u+c_{1}+\frac{c_{2}}{r} \\
\varphi^{\prime}(r)=-\frac{1}{r^{2}} \int_{0}^{r} u^{2} \int_{0}^{u} \frac{1}{s^{2}} \int_{0}^{s} t^{2} m(t) d t d s d u+c_{3} r
\end{gathered}
$$

with $c_{1}, c_{2}$ and $c_{3}$ constant and

$$
m(t)=\frac{\epsilon}{R^{3}} \chi_{(0, R)}(t) .
$$

If we take derivative in (118) and then we use Fubini's theorem we have

$$
\psi^{\prime \prime}(r)=\frac{1}{3 r^{3}} \int_{0}^{r} t^{3} h(t) d t+\frac{1}{3} \int_{r}^{\infty} h(t) d t-\frac{2 c_{2}}{r^{3}} .
$$

A similar manipulation gives us

$$
\varphi^{\prime \prime}(r)=-\int_{0}^{r} \frac{1}{s^{4}} \int_{0}^{s} t^{4} m(t) d t d s+c_{3} .
$$

In order to have $\psi^{\prime \prime} \geq 0$ and $\varphi^{\prime \prime} \geq 0$, we take in the above expressions $c_{2}=0$ and

$$
c_{3}=\int_{0}^{\infty} \frac{1}{s^{4}} \int_{0}^{s} t^{4} m(t) d t d s .
$$


Then

$$
\psi^{\prime \prime}(r)=\frac{1}{3 r^{3}} \int_{0}^{r} t^{3} h(t) d t+\frac{1}{3} \int_{r}^{\infty} h(t) d t
$$

and

$$
\varphi^{\prime \prime}(r)=\int_{r}^{\infty} \frac{1}{s^{4}} \int_{0}^{s} t^{4} m(t) d t d s+c_{3}
$$

It is easy to check that $\varphi^{\prime}(0)=0$ and therefore

$$
\varphi^{\prime}(r)=\int_{0}^{r} \varphi^{\prime \prime}(u) d u .
$$

If we take in (118) $c_{1}=\alpha$ and we use (117)-(122), we can check (114), (115) and $\Phi^{\prime}(r) \geq \alpha$.

To see $\Phi^{\prime}(r) \leq \kappa$, since $\psi^{\prime \prime} \geq 0$, we have

$$
\Phi^{\prime}(r) \leq \alpha+\frac{1}{2} \int_{0}^{\infty} t h(t) d t+\varphi^{\prime}(r) .
$$

From (121) and (122) we have that

$$
\varphi^{\prime}(r) \leq \frac{\epsilon}{6}
$$

and $\Phi^{\prime}(r)<\kappa<\frac{1}{2}$ follows by (112).

\section{REFERENCES}

1. M. Arai, Absolute continuity of Hamiltonian operators with repulsive potentials, Publ. Res. Inst. Math. Sci. 7 (1971-1972) 621-635.

2. S. Agmon, L. Hörmander, Asymptotic properties of solutions of differential equations with simple characteristics, Journal d'Analyse Mathmatique, 30 (1976), 1-38.

3. J. A. Barceló, A. Ruiz and L. Vega, Some dispersive estimates for Schrödinger equations with repulsive potentials, J. Func. Anal. 236 (2006) 1-24.

4. P. Constantin, J. C. Saut, Local smoothing properties of dispersive equations, J. Amer. Math. Soc. 1 (1988) 413-419.

5. J. Duoandikoetxea, Fourier Analysis, Graduate Sudies in Math. V 29. AMS (2001).

6. I.Gasser; P. A.Markowich; B. Perthame, Dispersion and moment lemmas revisited. J. Differential Equations 156 (1999), no. 2, 254-281.

7. M. Golberg, L. Vega and N. Visciglia, Contraexamples of Strichartz inequalities for Schrödinger equations with repulsive potential, Int. Math. Res. Not.(2006) .

8. T. Ikebe, Y. Saito Limiting absorption method and absolute continuity for the Schrödinger operator, J. Math. Kyoto Univ. 12,3 (1972) 513-542.

9. A. Ionescu and W. Schlag, Agmon-Kato-Kuroda theorems for a large class of perturbations, Duke Math. Journal. 131,3 (2006) 397-440.

10. T. Kato, K. Yajima Some examples of smooth operators and the ssociated smoothing effect, Math. Phy. v.1,n.4 (1989) 481-496.

11. J. E. Lin and W. A. Straus Decay and scattering of solutions of a nonlinear Schrödinger equation, J. Functional Analysis 30, (1978) 245-263.

12. C. S. Morawetz, Time daeay for the non-linear Klein-Gordon equation, Pro. Roy. Soc. A306 (1968), 291-296.

13. B. Perthame and L. Vega, Morrey-Campanato Estimates for Helmholtz Equations, J. Func. Anal. 164 (1999) 340-355.

14. B. Perthame and L. Vega, Energy concentraction and Sommerfeld condition for Helmholtz equation with variable index at infinity, To appear in GAFA.

15. M. Reed and B. Simon, Methods of Modern Mathemathical Physics. V. III Scattering Theory and V. IV Analysis of Operators, Academic Press, 1978. 
16. I. Rodnianski, W. Schlag, Time decay for solutions of Schrödinger equation with rough and time-dependent potentials, Invent. Math. 155 (2004) 455-513.

17. F. Rellich, Darstelluung der eigenverte von $\delta u+\lambda u$ durch ein randintegral, Math. Z. 46 (1940) 635-646.

18. A. Ruiz and L. Vega, On local regularity of Schrödinger equations, Int. Math. Res. Not. 1 (1993) 13-17.

19. P. Sjöjolin, Regularity of solutions to the Schrödinger equations, Duke Math. J. 55 (1987) 699-715.

20. L. Vega, Schrödinger equation: Pointwise convergence to the initial data, Proc. Amer. Math. Soc. 102 (1988) 874-878.

21. L. Vega and N. Visciglia, Asymptotic lower bounds for a class of Schrödinger equations, To appear in Comm. in Math. Physics.

J. A. Barceló, ETSi de Caminos, Universidad Politécnica de Madrid, 28040, Madrid, SPAIN.

E-mail address: juanantonio.barcelo@upm.es

A. Ruiz, Departamento de Matemáticas, Universidad Autónoma de Madrid, 28049, Madrid, SPAIN.

E-mail address: alberto.ruiz@uam.es

L. Vega, Departamento de Matemáticas, Universidad del País Vasco, Apartado 644, 48080 Bilbao, Spain.

E-mail address: luis.vega@ehu.es

M. C. Vilela, Departamento de Matemática Aplicada, Universidad de Valladolid, Plaza Santa Eulalia 9 y 11, 40005 Segovia, Spain.

E-mail address: maricruz@dali.eis.uva.es 